\title{
PROPERTIES OF NONLINEAR DYNAMO WAVES
}

\author{
S. M. TOBIAS \\ JILA, University of Colorado, Boulder, Colorado, \\ CO 80309-0440, USA
}

(Received 31 December 1996: In final form 8 April 1997)

\begin{abstract}
Dynamo theory offers the most promising explanation of the generation of the sun's magnetic cycle. Mean field electrodynamics has provided the platform for linear and nonlinear models of solar dynamos. However the nonlinearities included are (necessarily) arbitrarily imposed in these models. This paper conducts a systematic survey of the role of nonlinearities in the dynamo process, by considering the behaviour of dynamo waves in the nonlinear regime. It is demonstrated that only by considering realistic nonlinearities that are non-local in space and time can modulation of the basic dynamo wave be achieved. Moreover this modulation is greatest when there is a large separation of timescales provided by including a low magnetic Prandil number in the equation for the velocity perturbations.
\end{abstract}

Keywords: Dynamo; nonlinear; modulation

\section{INTRODUCTION}

Magnetic activity is found in many astrophysical bodies on all lengthscales. It is now widely believed that stellar magnetic fields are generated by hydromagnetic dynamos in which the field is maintained against ohmic dissipation by the velocity fields within the star. Research into dynamo theory has received much attention in the recent past and many new approaches have recently been introduced. Specific attention has often focussed on the origin of the solar magnetic field, as the sun is the only star in which the magnetic field can be observed in detail. 
The basic eleven year solar cycle is modulated on a longer timescale. For approximately 70 years during the seventeenth century, the amplitude of magnetic activity was dramatically reduced (Eddy, 1976; Ribes and Nesme-Ribes, 1993). This minimum in activity (The Maunder Minimum) can also be detected in the abundance records of terrestrial ${ }^{14} \mathrm{C}$ and ${ }^{10} \mathrm{Be}$, with both records indicating the occurrence of several previous minima in magnetic activity (Stuiver, 1994; Beer et al., 1991)

Numerical studies of the generation of magnetic field in the sun have usually fallen into one of three categories (for more details see Weiss and Tobias, 1997). Numerical integration of the fully self-consistent dynamo equations (Gilman, 1983; Glatzmaier, 1985) demonstrate that dynamo cycles can be found. However these integrations are expensive to perform and are only feasible using over-simplified physics. Consequently, a number of alternative mean-field models have been studied (see e.g., Brandenburg et al., 1989; Kitchatinov et al., 1994) that attempt to give a greater insight into the temporal and spatial behaviour of nonlinear dynamo models. These models have met with varying degrees of success, producing modulated cycles and chaotic solutions, but the exact rôle of the various competing nonlinear mechanisms (e.g., quenching) is unclear. Moreover the modulation found in these models is different to the behaviour found in the sun. Modulation of the basic cycle is associated with large changes in the parity (symmetry) of solutions, whilst the sun undergoes significant change in the amplitude of oscillation with only very small changes in parity (Watari, 1996; Yau, 1988).

The third approach is to use the recent advances made in the field of nonlinear dynamics to describe the behaviour of dynamo models. To illustrate how various aspects of solar magnetic activity may arise, it is often necessary to study toy models. These systems are not predictive, but they do enable the generic properties common to fully selfconsistent dynamos and idealised mean field models to be easily understood (Weiss, 1993). This has been dubbed Astromathematics by Spiegel (1995). One approach is to concentrate on the alternation between active and quiescent periods. This behaviour, termed 'on-off intermittency' by Spiegel (1995), can be modelled in a number of ways. The first of these is aperiodic switching. This switching may rely on stochasticity [studied in detail in full PDE simulations by Choudhuri 
(1992) and Ossendrijver and Hoyng (1996)] which is only likely to be of importance if the dynamo is marginal or, more naturally, deterministic chaos (Platt, 1994; Platt et al., 1994; Spiegel, 1995). Indeed some models have been constructed that use both mechanisms (Schmitt et al., 1996). Another technique is to consider the symmetries inherent in the problem and to construct a model that relies on the interactions (and resonances) between modes with different symmetries. Knobloch and Landsberg (1996) find that for such a model two types of minima may be found. A complementary technique is to consider truncated representations of the mean-field dynamo equations. For example, Weiss et al. (1984) extended Parker's (1955, 1979) linear dynamo wave calculations to include nonlinear effects. They constructed a sixth order system of ordinary differential equations. This system, a complex generalisation of the Lorenz equations, yielded three successive Hopf bifurcations as the dynamo number $D$ was increased. Multiply-periodic solutions lay on a three-torus in phase space. As $D$ was increased further the torus was destroyed and aperiodic solutions were found. The model can be simplified to a fifthorder system whose bifurcation structure has been studied in detail (Jones et al., 1985). These low-order models are open to the criticism that any behaviour found may be an artifact of the severe approximations made in truncating the system. For a model to be of astrophysical relevance, any properties should remain robust under small changes in the approximations and assumptions made. Covas et al. (1997) investigated the structural stability of a 'dynamic $\alpha$-effect' dynamo based on a model proposed by Schmalz and Stix (1991). They demonstrated that small changes in the level of truncation of dynamic forcing could have drastic consequences on the results of integrations. A different viewpoint was adopted by Tobias et al. (1995). They considered a robust third-order model constructed using the results of normal form theory and reproduced the bifurcation sequence found in the truncated model of Weiss et al. (1984). Although such results are expected to be robust, it has still to be demonstrated that this bifurcation structure would continue to be found in more realistic (PDE) models.

To describe the dynamic behaviour of the dynamo it is vital first to understand the rôle of the nonlinear terms in the equations. In many mean-field models the nonlinear effect of the Lorentz force is included 
to limit the growth of the magnetic instability. The back-reaction of the magnetic field on the velocity is often parametrised in such models. The small-scale motions, which lead to the $\alpha$-effect and turbulent diffusivity, are sensitive to changes in the magnetic field strength (microdynamic quenching). An ad-hoc approach usually used in nonlinear mean-field models is to parametrise the action of the magnetic field on the trubulent motions by including $\alpha$-quenching (see e.g., Stix, 1972). For consistency the turbulent diffusivity should be treated in a similar manner, because both tensors are derived from the same turbulence as first noted by Roberts and Soward (1975). The exact form of the quenching of the $\alpha$-effect and turbulent diffusion is an open question requiring the investigation of sophisticated numerical models. What is clear however is that both of these effects should be suppressed by the action of the Lorentz force.

The Lorentz force must also modify the large-scale velocity (a macrodynamic nonlinearity). This has often been included in models by quenching the differential rotation ( $\omega$-quenching). More sophisticated models include the back-reaction of the small-scale magnetic field on the $\Lambda$-effect in the equation for the mean velocity field (e.g., Kitchatinov et al., 1994). However the most natural consequence of the Lorentz force is the velocity perturbation driven by the mean magnetic field. This macrodynamic process has been termed the "Malkus-Proctor effect" after the work of Malkus and Proctor (1975), where it was used as the nonlinearity in an $\alpha^{2}$-dynamo model. Because the inclusion of this nonlinearity requires the solution of another equation coupled to the dynamo equation it has not been as extensively studied as $\alpha$-or $\omega$-quenching.

In this paper the effects of including both the microdynamic quenching mechanisms and the Malkus-Proctor effect on the properties of dynamo waves are investigated. It is necessary to isolate the role of nonlinearities in the dynamo process from other effects that may be of importance in dynamo models. For example boundary conditions (Worledge et al., 1997 Tobias et al., 1997) and inhomogeneities (Meunier et al., 1997) can both play a crucial rôle in determining the nature of solutions. The consequence of imposing various nonlinearities on wave-like solutions should therefore be determined before an attempt is made to include them in more realistic models. In this paper the simplest physically relevant model of a solar dynamo is considered. 
The model is an extension of the one proposed by Parker (1993) for a dynamo acting at the base of the convection zone and characterised by a layer of strong shear lying beneath a layer where the $\alpha$-effect is strong. In the Section 2 the important results of Parker are summarised and the features which are to be included in the new model are introduced. In Section 3 the new dynamo model is derived from the $\alpha \omega$ dynamo equations, and the linear theory for the new model is included in Section 4. Simple nonlinear behaviour is discussed in Section 4 where the consequences of including (microscopic) quenching mechanisms in the model are outlined. It is demonstrated that $\eta$-quenching plays a very different role to simple equilibrating mechanisms such as $\alpha$-quenching. The most important results are contained in Section 5 where the "Malkus-Proctor effect" is introduced and interesting temporal behaviour can be found. The bifurcation structure of the system is analysed and related to that found in earlier models. A discussion of the consequences of the results for solar and stellar dynamo theory is then included in Section 6.

\section{THE PARKER SURFACE DYNAMO WAVE}

The linear model considered by Parker (1993) demonstrates how a dynamo at the interface of the solar convection zone and radiative interior might work. The model provides the motivation this paper and, for this reason, a brief summary of the set-up and results for this system is included in this section.

Parker constructed an extension of his initial (1955) 'Dynamo Wave" model in order to take into account the available observational data. A model of the base of the convection zone and the overshoot region which lies below is considered. Helioseismology (e.g., Thompson et al., 1996) indicates that there is a thin layer of radial shear at the base of the convection zone. This region of strong shear in the tachocline makes it a good candidate for the site of production of toroidal flux. However magnetic buoyancy of this strong azimuthal field needs to be suppressed in order to prevent the flux escaping to the surface on a timescale too short for the effective operation of a dynamo. How might the confinement of the toroidal field to this region be achieved? Parker argues that the total amount of azimuthal flux beneath active 
latitudes is $10^{23}-10^{24}$ Maxwells. If all this flux were to be concentrated into a thin region at the base of the convection zone the field intensity would need to be of the order of 2.5 kilogauss or more. The strong field would act back on the turbulence, suppressing the eddy diffusivity (via a nonlinear mechanism) and confining the field to a thin region. The problem is now that a field of this strength would also quench the $\alpha$-effect, meaning that there would now be no generation of poloidal field in this region. The solution is to notice that, if the field is strongly confined, the suppression of the turbulent diffusivity and $\alpha$-effect is local. The toroidal field in the bulk of the convection zone is weak and hence the cyclonic turbulence would only be weakly modified. Indeed Charbonneau and MacGregor (1996) have shown that this type of interface dynamo can work even if the $\alpha$-effect is as strongly suppressed as has recently been suggested (Vainshtein and Cattaneo, 1992; Cattaneo and Hughes, 1996). The convection zone is therefore the favoured site for production of poloidal flux from toroidal flux and the dynamo cycle is completed. The toroidal flux generated by the strong shear in the tachocline diffuses slowly into the convection zone where it is converted by the cyclonic turbulence into poloidal flux. This flux may then diffuse back to the overshoot region where it can act as a source for production of toroidal field. Here the region of generation of toroidal and poloidal fields are separated in space and the dynamo has to rely on diffusion to operate. For this reason it is less efficient than models which have the $\alpha$-effect and $\omega$-effect in the same location. Other models with the generating regions in different places (e.g., Steenbeck and Krause, 1969; Kleorin and Ruzmaikin 1981) have previously been proposed.

The model is constructed as follows. Parker considers local Cartesian coordinates with the origin at the base of the convection zone [as shown in Fig. l(a)]. The system is two-dimensional and the surface $z=0$ is the upper boundary of a region $(z<0)$ of uniform shear, $G=d v_{0} / d z$, where the eddy diffusivity is reduced to a value $n$ and there is no $\alpha$-effect. The region $z>0$ is convective and hence has a large turbulent diffusivity $(\eta)$ and a strong $\alpha$-effect, but no shear; a diagram of the cartesian model is shown in Figure 1(b). Parker considers planewave solutions proportional to $e^{\sigma t}$ to the linear dynamo equations in the 2 layers, and the critical parameter is the non-dimensional dynamo number $D=G \alpha / \eta^{2} k^{3}$ where $k$ is the wavelength of the solution in the 


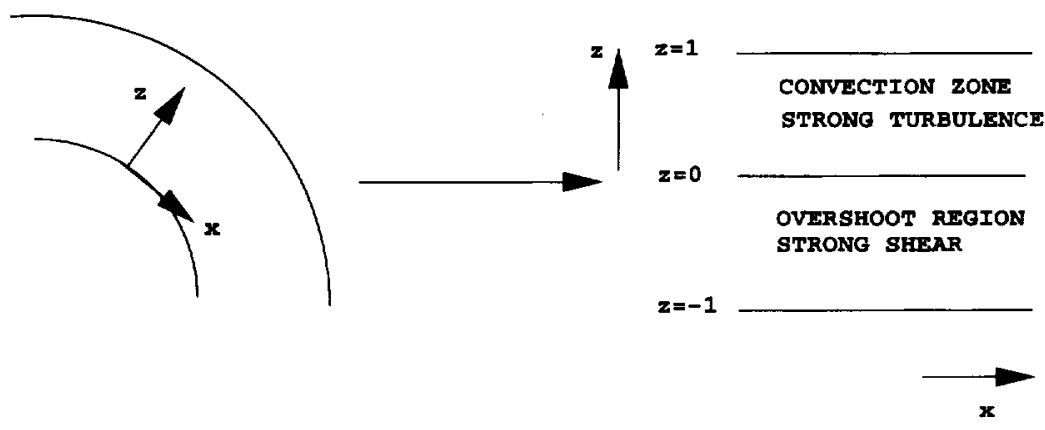

FIGURE I The local Cartesian coordinates used by Parker (1993).

$x$-direction. For $D<D_{\text {crit }}$ all plane wave solutions decay away as $t \rightarrow \infty$. However for $D>D_{\text {crit }}, \mathbf{R}(\sigma)>0$ and exponentially growing solutions may be found. Parker examines two cases in detail. The first is when the ratio of the diffusivities in the two layers is unity so $\mu^{2} \equiv n / \eta=1$. In this case the dispersion relation that determines $\sigma$ reduces to a simple form and $D_{\text {crit }}$ may easily be found. This simple calculation establishes the existence and nature of the solutions. They take the form of a dynamo surface wave propagating along the interface between the region of cyclonic convection and the region of shear. The second case considered is the limit $n \ll \eta$ so that $\mu^{2} \ll 1$. This represents the situation of very small flux transport in the region of differential rotation $(z<0)$. Here the calculation yields a vertical scale for the toroidal field in $z<0$ that is substantially smaller than that in $z>0$ and the azimuthal field in $z<0$ is confined to a thin layer pressed up against the underside of $z=0$. The field in $z>0$ is more diffuse. Further calculation yields the interesting result that in order for the calculation to be consistent, the relationship

$$
n b_{\max }^{2} \propto \eta B_{\max }^{2}
$$

must hold where $b_{\max }$ and $B_{\max }$ are the maximum values of the toroidal field in the regions $z<0$ and $z>0$ respectively. The relationship in (1) is kinematic, and for a given $\mu^{2}$ determines the ratio $b_{\max }^{2} / B_{\max }^{2}$. This scenario is therefore entirely self-consistent if the eddy-diffusivity is suppressed inversely with the magnetic energy density. 
Because the calculation is linear it does not contain any information about how the magnetic field may act in the nonlinear regime. Parker hypothesised that some form of dynamical balance is achieved in the sun, and used the example of the action of Maxwell stresses on Alfvén waves to illustrate a possibility for this balance. In this paper we examine how the addition of nonlinearities changes the surface wave model, and which properties of the linear model remain robust in the nonlinear regime. In the next section, the nonlinear dynamo problem is discussed and the results of previous nonlinear models are presented.

\section{DERIVATION OF THE MODEL}

The starting point of the derivation is the mean field dynamo equation

$$
\frac{\partial \mathbf{B}}{\partial t}=\nabla \times(\mathbf{u} \times \mathbf{B}+\alpha \mathbf{B}-\eta \nabla \times \mathbf{B}),
$$

where $\mathbf{B}$ and $\mathbf{u}$ are the mean magnetic field and velocity field of the plasma respectively. The turbulent diffusivity $(\eta)$ and the $\alpha$-effect arise from the mean interaction of the small-scale fluctuating magnetic and velocity fields. The dynamo equations are considered in the twodimensional Cartesian coordinates considered by Parker. Immediate progress can be made by decomposing the magnetic field into toroidal and poloidal parts, i.e.

$$
\mathbf{B}=B(x, z) \hat{\mathbf{y}}+\nabla \times[\mathbf{A}(x, z) \hat{\mathbf{y}}] .
$$

Equation (2) is clearly linear in the magnetic field $\mathbf{B}$ and for a given velocity field solutions are either exponentially growing or decaying. As noted in the introduction, nonlinearities may be introduced in the model by parameterizing the back-reaction of the magnetic field on the turbulence ( $\alpha$ and $\eta$-quenching).

In order to include the effect of the magnetic field in driving a velocity perturbations (macrodynamic nonlinearities) it is necessary to consider the equation for the large-scale velocity $\mathbf{u}$. The momentum equation for the mean velocity field $\mathbf{u}$ is given by

$$
\rho\left[\frac{\partial \mathbf{u}}{\partial t}+(\mathbf{u} \cdot \nabla) \mathbf{u}\right]=-\nabla p+\nabla \cdot \boldsymbol{\sigma}+2 \boldsymbol{\Omega} \times \mathbf{u}+\mathbf{j} \times \mathbf{B}+\nabla \cdot \mathscr{F},
$$


where $\rho$ is the density, $p$ is the pressure $\Omega$ is the rotation of the star, $\sigma$ is the stress tensor and $\mathbf{j}=1 / \mu_{0} \nabla \times \mathbf{B}$ is the large-scale current. The effects of rotation and magnetic field on the velocity are clear in (4), and the other forces (e.g., gravity, thermal forcing, Reynolds stresses from the small-scale turbulence) are contained in the tensor, $\mathscr{\mathcal { F }}$.

Progress is made by assuming that the large-scale velocity consists of two separate components. The first component would be present in the absence of a magnetic field, and is generated by purely hydrodynamic effects. This velocity is driven by rotational, gravitational and thermal effects and provides the basic state of the model. The second component arises owing to the back-reaction of the magnetic field via the Lorentz force. It is important to stress here that the Lorentz force generated by the dynamo will drive a meridional flow in addition to the toroidal velocity considered here. Here we ignore this effect and concentrate solely on the toroidal velocity perturbations. It will be interesting in the future to study models where the latitudinal shear is also included and to investigate the interaction of the driven meridional flow with this shear.

In the model, the above statements correspond to setting

$$
\mathbf{u}=\mathbf{U}+\mathbf{v}=U(x, z) \hat{\mathbf{y}}+v(x, z) \hat{\mathbf{y}},
$$

where $U$ is the imposed basic large-scale velocity [given by $d U / d z=G(z) ; G(z)$ is the shear defined by the Parker model] and $v$ is the velocity perturbation that is driven by the macroscopic magnetic stresses due to the Malkus-Proctor effect.

In the calculations that follow, the vertical extent of the layer is considered to be small compared to a density scale height and therefore the density $\rho$ is set to be a constant. This is a reasonable assumption for a dynamo located in a thin region at the base of the solar convection zone, where the density scale height is large. Here we consider solutions of the coupled system (2) $-(5)$. These equations are non-dimensionalised by setting

$$
\mathbf{x}=L \hat{\mathbf{x}}, t=L^{2} / \eta_{0} \hat{t}, \quad \eta=\eta_{0} h(z), \quad \alpha=\alpha_{0} f(z), \quad G(z)=\omega_{0} g(z),
$$

$$
v(z)=L \omega_{0} \hat{v}(z), \quad A(z)=L B_{0} \alpha_{0} \hat{A}(x, z), \quad B(x, z)=B_{0} \hat{B}(x, z),
$$


where $L$ is a characteristic lengthscale in the $z$-direction. In the limit where the shear is strong in comparison with the $\alpha$-effect (the $\alpha \omega$ limit) the following equations are obtained

$$
\begin{gathered}
\frac{\partial A}{\partial t}=h\left[\frac{\partial^{2} A}{\partial x^{2}}+\frac{\partial^{2} A}{\partial z^{2}}\right]+f B \\
\frac{\partial B}{\partial t}=h\left[\frac{\partial^{2} B}{\partial x^{2}}+\frac{\partial^{2} B}{\partial z^{2}}\right]+\frac{\partial h}{\partial z} \frac{\partial B}{\partial z}+D\left[g(z)+\frac{\partial v}{\partial z}\right] \frac{\partial A}{\partial x}-D \frac{\partial v}{\partial x} \frac{\partial A}{\partial z} \\
\frac{\partial v}{\partial t}=\tau h\left[\frac{\partial^{2} v}{\partial x^{2}}+\frac{\partial^{2} v}{\partial z^{2}}\right]+\tau \frac{\partial h}{\partial z} \frac{\partial v}{\partial z}+\Upsilon\left[\frac{\partial B}{\partial z} \frac{\partial A}{\partial x}-\frac{\partial A}{\partial z} \frac{\partial B}{\partial x}\right]
\end{gathered}
$$

where $\tau=\nu_{0} / \eta_{0}$ (the magnetic Prandtl Number) is assumed to be a constant and

$$
D=\frac{\alpha_{0} \omega_{0} L^{3}}{\eta_{0}^{2}}, \Upsilon=\frac{B_{0}^{2} \alpha_{0} L}{\rho_{0} \mu_{0} \eta_{0}^{2} \omega_{0}}
$$

Here $D$, the non-dimensional dynamo number, is a measure of the efficiency of the dynamo giving the strengths of the $\alpha$ and $\omega$ effects. The parameter $\Upsilon$ is therefore the product of the " $\alpha$-effect Reynolds number' $R_{\alpha}=\alpha_{0} L / \eta_{0}$ and the (modified) Elsasser Number $\Lambda=B_{0}^{2} / \rho_{0} \mu_{0} \eta_{0} \omega_{0}$. It is important to note at this point that, because this parameter contains the quotient $\alpha_{0} / \omega_{0}$, the sign of $\Upsilon$ is the same as that of the dynamo number $D$, i.e. if the dynamo number is chosen to be negative because the product $R_{\alpha} R_{\omega}$ is negative then for consistency the parameter $\Upsilon$ is negative also.

Equation (6) is to be solved in a two-dimensional layer $-1<z<1$, $0<x<x_{\max }$ for suitable choices of the functions $f, g$, and $h$. Here we set

$$
\left.\begin{array}{c}
f(z, B)= \\
\hat{f}(z) Q_{\sigma}(B)
\end{array}\right\}= \begin{cases}\frac{1}{2}\left[1+\tanh E\left(z-z_{3}\right)\right]\left[1-e^{-25\left(z-z_{0}\right)^{2}}\right] Q_{\sigma}(B), & z>z_{0}, \\
0, & z<z_{0},\end{cases}
$$

$$
\left.\begin{array}{l}
g(z, B)= \\
\hat{g}(z) Q_{\kappa}(B)
\end{array}\right\}= \begin{cases}\frac{1}{2}\left[1-\tanh E\left(z-z_{2}\right)\right]\left[1-e^{-25\left(z-z_{1}\right)^{2}}\right] Q_{\kappa}(B), & z<z_{1} \\
0, & z>z_{1}\end{cases}
$$




$$
\left.\begin{array}{c}
h(z, B)-\eta_{0}= \\
\hat{h}(z) Q_{\mu}(B)
\end{array}\right\}= \begin{cases}\frac{1}{2}\left[1+\tanh E\left(z-z_{3}\right)\right]\left[1-e^{-25\left(z-z_{0}\right)^{2}}\right] Q_{\mu}(B), & z>z_{0}, \\
\eta_{0}, & z<z_{0},\end{cases}
$$

where $E, z_{0}, z_{1}, z_{2}, z_{3}$ are all real parameters that may in principle be varied. The nonlinear quenching mechanisms to be employed are given by $Q_{\sigma}(B), Q_{\kappa}(B)^{\prime}$ and $Q_{\mu}(B)$, with the level of quenching depending on the value of $\sigma, \kappa$ and $\mu$ respectively. Both local and global (where the quenching is inversely proportional to the average energy over the period of a wave) quenching laws will be investigated.

It is difficult to visualise the form of the $\alpha$-effect, shear and turbulent diffusion from the mathematical formulae (8)-(10), and so an example of the imposed profiles for the $\alpha$-effect, $\omega$-effect and turbulent diffusivity for a particular choice of parameters is shown in Figure 2. This figure shows that the profiles are similar to those considered by Parker in his linear model (shown in Fig. 1). The $\alpha$-effect is limited to the convection zone $(z>0)$ and the shear is strongest in the overshoot

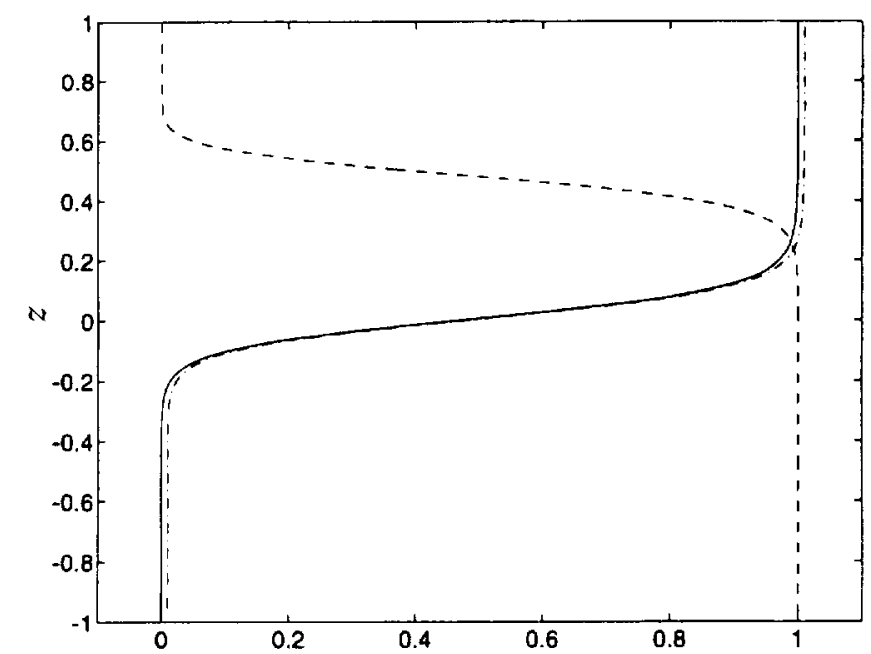

FIGURE 2 Profiles for the shear (dashed), alpha-effect (solid) and diffusivity (dotdashed) showing the $z$-dependence. Here $z_{0}=-0.75, z_{1}=0.0$ and $z_{3}=0.75$ are fixed. The parameters chosen are $E=10, z_{2}-0.5$. Notice the region of overlap of the $\alpha$-effect and shear. 
region $(z<0)$. The dependence of solutions on the details of these profiles is discussed below.

\section{LINEAR THEORY}

\subsection{Derivation of Equations and Boundary Conditions}

The aim of the linear calculation is to determine the critical dynamo number, i.e., the value of $D$ at which instability to dynamo action sets in. The perturbed velocity $v$ only arises as a result of nonlinear interactions, and so for the linear magnetic theory $v=0$. Moreover the nonlinear quenching is not considered and so $Q_{\sigma}=Q_{\kappa}=Q_{\mu}=1$. In light of the work of Parker $(1955,1993)$ the initial bifurcation is expected to be a Hopf bifurcation to travelling wave solutions; the preferred direction of travel depends on the sign of the dynamo number. Travelling wave solutions of the form $A \equiv a(z) e^{i k x+\sigma t}$, $B \equiv b(z) e^{i k x+\sigma t}$ are sought where $\sigma=\lambda+i \omega$. Thus the dynamo waves have frequency $\omega$ and have a growth-rate $\lambda$. the problem now reduces to finding the value of $D=D_{c}$ for which $\lambda=0$. We set $a(z)=a_{R}(z)+$ $i a_{I}(z), b(z)=b_{R}(z)+i b_{l}(z)$, substitute into (6) and equate real and imaginary parts to obtain the set of coupled ODEs

$$
\begin{aligned}
& h(z) h_{R}^{\prime \prime}(z)+h^{\prime}(z) b_{R}^{\prime}(z)-\left[h(z) k^{2}+\lambda\right] h_{R}(z)=-\omega h_{I}+k D g(z) a_{l}, \\
& h(z) b_{I}^{\prime \prime}(z)+ h^{\prime}(z) b_{l}^{\prime}(z)-\left[h(z) k^{2}+\lambda\right] h_{I}(z)=\omega b_{R}-k D g(z) a_{R}, \\
& h(z) a_{R}^{\prime \prime}(z)-\left[h(z) k^{2}+\lambda\right] a_{R}(z)=-\omega a_{I}-f(z) b_{R}, \\
& h(z) a_{I}^{\prime \prime}(z)-\left[h(z) k^{2}+\lambda\right] a_{l}(z)=\omega a_{R}-f(z) b_{l} .
\end{aligned}
$$

Initially the boundary conditions

$$
A=B=0 \text { at } z=-1, \quad \frac{\partial A}{\partial z}=B=0 \text { at } z=1
$$

are chosen as reasonable conditions at the bottom and top of the layer. Physically these conditions correspond to setting $\mathbf{B}_{y}=\mathbf{B}_{x}=0$ at the top boundary and $\mathbf{B}=0$ at $z=-1$. It is desirable to show that changing these boundary conditions has only a weak effect on the main properties of the solution. The linear solutions are periodic in $x$ with 
period $2 \pi / k$. The linear theory will determine the preferred scale (i.e., wavenumber) in the $x$-direction and this will be used in the nonlinear regime to select the aspect ratio of the box. The boundary conditions imply that

$$
\begin{array}{ll}
b_{R}=b_{I}=a_{R}=a_{I}=0, & z=-1 \\
b_{R}=b_{I}=a_{R}^{\prime}=a_{I}^{\prime}=0, & z=+1
\end{array}
$$

The equations and boundary conditions constitute a two-point boundary value eigenvalue problem that may be solved numerically. Two numerical schemes were employed, the first used the program NRK (see Gough et al., 1976) which employs the Newton-RaphsonKantorovitch method using a second order centred-difference discretisation. The results were checked using the NAG routine $\mathrm{D} 02 \mathrm{HBF}$, which uses a shooting method to solve the eigenvalue problem.

\subsection{Properties of Eigensolutions}

The profiles shown in Figure 2 are selected as a realistic scenario for the base of the convection zone. They are a slight modification of the model proposed by Parker (1993). It seems likely that the transition between the region dominated by shear and that in which there is an efficient $\alpha$-effect will be fairly sharp, but that this will be a smooth transition (rather than a discontinuous jump). Moreover, it would be surprising if there were no overlap of these two regions, as the convection may overshoot into the region of shear (and indeed the shear may persist slightly into the base of the convection zone). It will be shown later that the important properties of the solutions are unaffected by the precise choice of profile, and it is only the form of the eigenfunctions that change significantly.

There is the symmetry $\mathrm{D} \rightarrow-D, x \rightarrow-x$ inherent in the problem, and for this reason only the case $D<0$ need be considered. The results for $D>0$ are the same but with the direction of travel of the dynamo wave reversed. To determine the preferred scale in the $x$-direction, the critical dynamo number for a given horizontal wavenumber $k$ is calculated. The calculation is then repeated for other values of $k$ and the curve of marginal stability is shown in Figure 3. The curve has a 

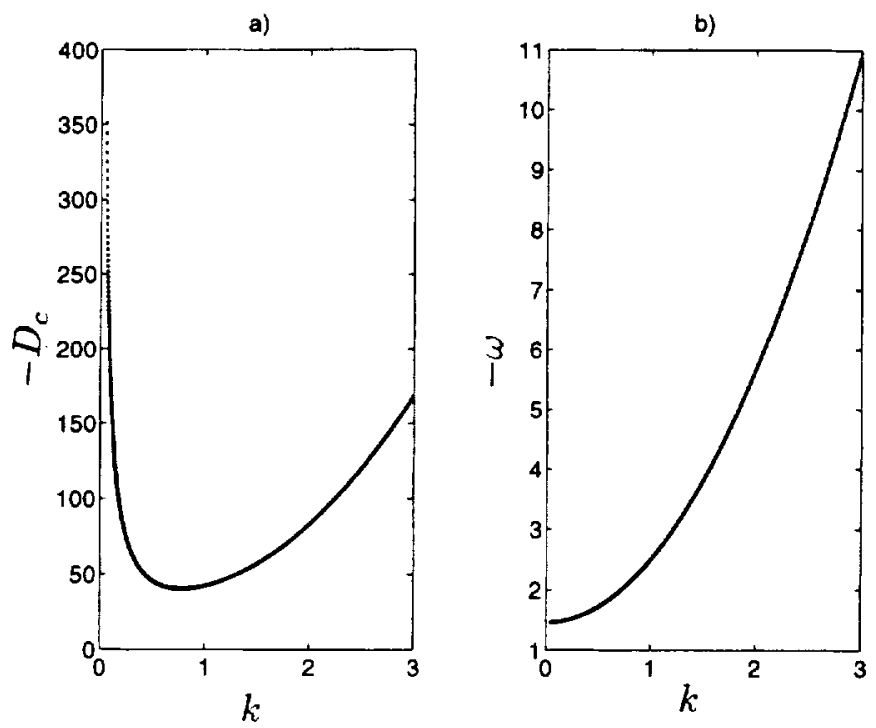

FIGURE 3 (a) Graph of critical dynamo number, $-D_{c}$, against wavenumber, $k$, for the parameter values chosen in Figure 2. THe curve has a minimum and therefore there exists a preferred lengthscale for the problem. (b) Graph of frequency $\omega$ against wavenumber, $k$ for the same parameter values as in (a) (with $D=D_{c}$ ). The frequency is a monotonically increasing function of wave number and tends to a limit as $k \rightarrow 0$.

minimum, and therefore a preferred scale. The minimum dynamo number is $\left|D_{c}\right|=-D_{c}^{*}=40.24$ and this occurs at $k=k^{*}=0.78$. This value suggests that it is sensible to consider the region $0<x<2 \pi$ in the nonlinear regime which corresponds to a wavenumber $k=1$ for which the critical dynamo number is $D_{c}=-42.0$. In a similar way the second eigenvalue, corresponding to the frequency $\omega$ of the travelling wave at the onset of dynamo action, can also be calculated as a function of wavenumber, and this curve is shown in Figure 3(b). Here $\omega$ is a monotonic function of wavenumber tending to infinity as $k$ is increased and to a finite limit, $\omega \rightarrow 1.46$ as $k \rightarrow 0$. For $k=1$ the frequency is $\omega=2.50$, and so the period of the wave is given by $T=$ $2 \pi /|\omega|=2.51$.

A contour plot of one wavelength of the eigenfunction with $k=1$ is shown in Figure 4(a,c). The toroidal field is generated by the shear in the region $z<0.5$ and diffuses to the region of $\alpha$-effect $(z>0)$ where the poloidal field is regenerated. For this reason the bulk of the toroidal 

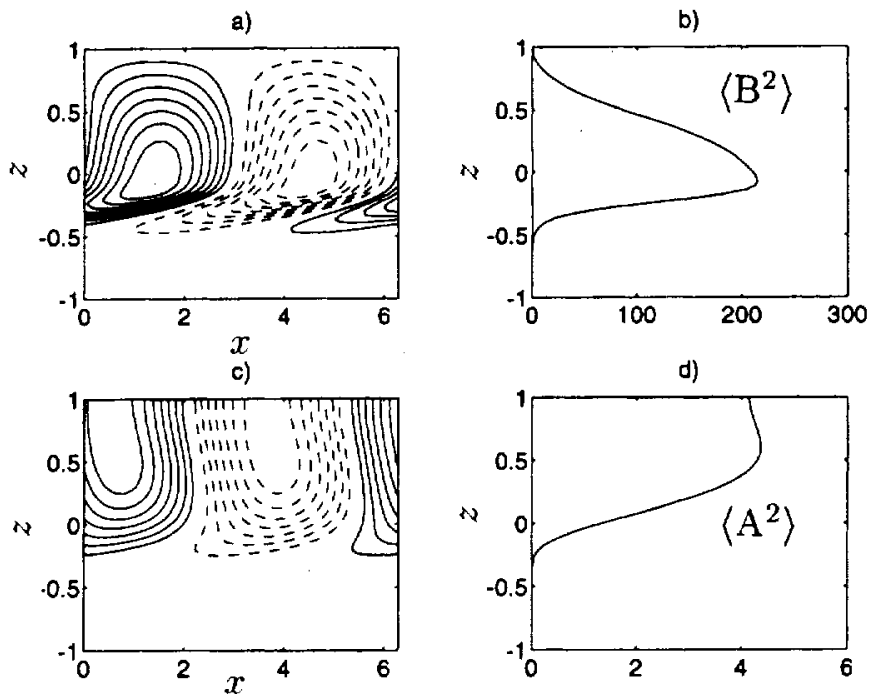

FIGURE 4 (a) One wavelength of the eigenfunction for the toroidal field $(B)$ in the linear problem, with parameters chosen as in Figure 2. (b) Graph of $\left\langle B^{2}\right\rangle$ versus $z$, which clearly shows the $z$-dependence of the linear eigenfunction. The toroidal field is generated by the $\omega$-effect from the $\alpha$-effect and is strongest in, and just below, the region of overlap of the $\alpha$-effect and $\omega$-effect. (c) As (a) but showing contours of the vector potential A. (d) As (b) but for $\left\langle A^{2}\right\rangle$ versus $z$. The graph shows that $\left\langle A^{2}\right\rangle /\left\langle B^{2}\right\rangle$ is small (although only the relative size of the fields is determined by the linear theory).

field is generated and stored in (and just below) the region of overlap of $\alpha$-effect and shear. Similarly the poloidal field generated by the $\alpha$-effect from the toroidal field lies in $z>0$, in (and just above) the overlap region as in Figure $4(b, d)$ where the $z$-dependence of the eigen functions is shown. These results are consistent with those found by Parker (1993) for his linear model.

The calculations were then repeated for values of the parameters that change the shape of the profiles for the $\alpha$-effect, shear and diffusivity, given in equations $(8)-(10)$. These results are summarised in Table I. As expected, the larger the region of overlap of the shear and $\alpha$-effect the more efficient the dynamo; therefore the critical dynamo number is smaller for profiles that have large regions of overlap. It is also apparent that the preferred wavenumber does not vary significantly when the parameters are changed. All the calculations undertaken have $k=1$ as the preferred integer wavenumber. This 
TABLE 1 This table shows the minimum critical dynamo numbers, $-D_{r}^{*}$, and the corresponding wavenumbers, $k^{*}$, for various parameter choices for the linear problem detined in (11) and (13). Also shown are the values of the critical dynamo numbers and frequencies for $k=1$. These will be the critical dynamo numbers in the nonlinear problem. It is clear, that although changing the profiles has a qualitative effect on these values, the critical wavenumbers and frequencies are all similar. The critical values for $E-10, z_{2}=0.5$ are shown in bold type. These are the parameters that will be used in the nonlinear theory

\begin{tabular}{rlrccc}
\hline \multicolumn{5}{c}{ Effect of Changing $\alpha, \omega$ and $\eta$ profiles } \\
$E$ & $z_{2}$ & $-D_{c}^{*}$ & $k^{*}$ & $-D$ at $k=1$ & wat $k=1$ \\
\hline 1 & 0.5 & 9.59 & 0.58 & 12.0 & 1.07 \\
$\mathbf{1 0}$ & $\mathbf{0 . 5}$ & $\mathbf{4 0 . 2 4}$ & $\mathbf{0 . 7 8}$ & $\mathbf{4 2 . 0}$ & $\mathbf{2 . 5 0}$ \\
20 & 0.5 & 55.19 & 0.83 & 56.7 & 3.02 \\
50 & 0.5 & 67.33 & 0.87 & 69.1 & 3.65 \\
1 & 0 & 12.97 & 0.58 & 16.3 & 1.03 \\
10 & 0 & 110.0 & 0.75 & 116.9 & 1.41 \\
\hline
\end{tabular}

would seem to indicate that the chosen scale for the eigenfunction arises not from any lengthscale that may be introduced by the profiles (i.e., the length of the overlap region or a typical length for the switchoff of the $\alpha$-effect), but from the nature of the Parker model and the radial extent of the domain.

The frequency is a measure of the efficiency of dynamo action. In the linear regime a more effective measure of the efficiency is the growth-rate of the solutions (and consequently the critical dynamo number), but in the nonlinear regime where solutions grow until equilibration a useful measure of efficiency is how quickly the dynamo can generate field of the opposite sign (i.e., how quickly the field can reverse) and this is given by the frequency of oscillation. As noted by Parker (1993), a dynamo with regions of $\alpha$-effect and shear separated in space is less efficient than one where the poloidal and toroidal fields are generated in the same location, because of the time taken for the toroidal field produced by the shear to diffuse to the region where the poloidal field is generated (and vice-versa). Larger separation of the two regions implies less efficient dynamo action, and it should therefore also be the case that the dynamo is made less efficient if the diffusivity between the two regions is reduced - the magnetic field then takes longer to diffuse between them. This explains the changes in the frequency shown in Table I - the frequency is higher (indicating a 
more efficient dynamo) for profiles that have a larger region of overlap and a larger turbulent diffusivity in that region. This explanation will be useful in analysing the nonlinear results when the back-reaction of the magnetic field on the profiles (and hence the frequency) is considered.

The effects of changing the boundary conditions of the linear problem and introducing anisotropies into the turbulent diffusion on the nature of the solutions are now investigated. Solutions are found for the same equations with modified boundary conditions on $A$ at the top of the box, so that (11) is now solved subject to the boundary conditions

$$
\begin{array}{ll}
b_{R}=b_{I}=a_{R}=a_{I}=0, & z=-1, \\
b_{R}=b_{I}=a_{R}=a_{I}=0, & z=+1 .
\end{array}
$$

The parameter values defining the profiles for the shear, $\alpha$-effect and turbulent diffusivity were chosen to be the same as in the first calculation. It is found that there are no qualitative differences in the curves of marginal stability - the only differences are quantitative. For this calculation $D_{c}^{*}=-74.05$ and this occurs at $k=k^{*}=1.09$. Clearly $k=1$ will again be the preferred mode in the nonlinear calculation. (The slight increase in the size of the critical dynamo number can be attributed to the fact that the $A=0$ boundary condition at $z=1$ makes poloidal fields harder to excite near the boundary.) Also the frequency is as before a monotonic function of wavenumber.

\subsection{Summary of Linear Results}

The dependence of critical dynamo numbers and wavespeed on wavenumber has been calculated for a variety of parameter values and boundary conditions. The results clearly show that the preferred lengthscale is not determined by the precise choice of parameters in the model and that other properties of the solution are robust and are not sensitive to the choice of either profile or boundary conditions. Rather they are determined by the general characteristics of the model (large turbulent diffusivity, shear and $\alpha$-effect largely separated). Therefore, these properties should also apply in the nonlinear regime and indeed in more elaborate systems based on the same model. 


\section{QUENCHING MECHANISMS}

In this section the consequences of including simple quenching mechanisms in the model are investigated and a systematic survey of these types of nonlinearities is undertaken. The most usual quenching mechanism employed in dynamo models is $\alpha$-quenching where the magnetic field acts back on the small-scale helical motions. It has been argued (e.g., Vainshtein and Cattaneo 1992; Cattaneo and Hughes 1996) that this effect can lead to suppression of the growth of magnetic field at levels much lower than equipartition. In this section no assumptions are made $a$ priori about the strength of this effect - rather it is considered to be a parameter of the model. Similarly $w$-quenching and $\eta$-quenching (representing modification of the angular momentum profile and turbulent diffusion by the magnetic field) are considered.

\subsection{Non-Local Quenching}

The simplest extension of the model to the non-linear regime is to consider simple quenching mechanisms acting in isolation. In the past these have been the preferred non-linearities in many dynamo models, and so the results should act as a benchmark for the more complicated non-linearities considered later. Here the Malkus-Proctor effect is ignored by setting $\Upsilon=0$ in (6c). Now (6c) decouples and (6a, 6b) may be solved in isolation with $v$ set to be zero.

The most basic forms of quenching to consider are those where the $\alpha$-effect, $\omega$-effect and turbulent diffusion are quenched inversely with the average magnetic energy of the wave. That is we set

$$
Q_{\sigma}=\frac{1}{1+\sigma \overline{B^{2}}}, \quad Q_{\kappa}=\frac{1}{1+\kappa \overline{B^{2}}}, \quad Q_{\mu}=\frac{1}{1+\mu \overline{B^{2}}},
$$

where again an overbar indicates a horizontal average. As discussed extensively in Tobias (1996b), the nature of the solution inherent in the choice of periodic boundary conditions makes this form of quenching a natural approximation. Local (in both $x$ and $z$ ) quenching mechanisms will give qualitatively similar results as discussed in Section (5.2). 
Now the $\alpha$ and $\omega$ effects and turbulent diffusion are not only functions of position but are also modified by the average toroidal field $\overline{B^{2}}$. Nonlinear travelling wave solutions of the form

$$
\begin{aligned}
& B=\sum_{j=1}^{m} b_{j}^{s}(z) \sin j(x-c t)+b_{j}^{c}(z) \cos j(x-c t), \\
& A=\sum_{j=1}^{m} a_{j}^{s}(z) \sin j(x-c t)+a_{j}^{c}(z) \cos j(x-c t),
\end{aligned}
$$

can be found using a continuation method. Here $c$ is the speed of propagation of the wave in the $x$-direction and the wavenumber is set to unity. The Galerkin expansion is now substituted into $(6 \mathrm{a}, 6 \mathrm{~b})$ to yield the system comprising the $4 \mathrm{~m}$ coupled nonlinear ordinary differential equations for the coefficients; i.e., for $j=1, m$

$$
\begin{aligned}
h\left[b_{j}^{\prime \prime c}(z)-j^{2} b_{j}^{c}(z)\right]+c j b_{j}^{s}(z)+D g j a_{j}^{s}(z)+h^{\prime} b_{j}^{c c}(z) & =0, \\
h\left[b_{j}^{\prime \prime s}(z)-j^{2} b_{j}^{s}(z)\right]-c j b_{j}^{c}(z)-D g j a_{j}^{c}(z)+h^{\prime} b^{\prime} c_{j}(z) & =0, \\
h\left[a_{j}^{\prime \prime c}(z)-j^{2} a_{j}^{c}(z)\right]+c j a_{j}^{s}(z)+f b_{j}^{c}(z) & =0, \\
h\left[a_{j}^{\prime \prime s}(z)-j^{2} a_{j}^{s}(z)\right]-c j a_{j}^{c}(z)+f b_{j}^{s}(z) & =0,
\end{aligned}
$$

where' denotes differentiation with respect to $z$. At this point it is important to recall that $h, g$, and $f$ are all functions of both $z$ and $\overline{B^{2}}$ and hence there is coupling between all the modes. This is apparent when it is noted that Parseval's Theorem implies

$\overline{B^{2}}(z)=\sum_{j=1}^{m}\left[b_{j}^{c}(z)^{2}+b_{j}^{s}(z)^{2}\right], \quad \frac{d \overline{B^{2}}}{d z}(z)=2 \sum_{j=1}^{m}\left[b_{j}^{c}(z) b_{j}^{\prime c}(z)+b_{j}^{s}(z) b_{j}^{\prime s}(z)\right]$,

and so all the modes in the sum appear in every equation in (17).

Equation (17) defines a new two-point boundary value problem, with the same boundary conditions as those initially considered in the linear theory (solutions are implicitly periodic at $x=0,2 \pi$ ). In this way both stable and unstable branches of travelling wave solutions to the problem may be located. 
The stability of these solution branches is then determined by timestepping the equations using a pseudo-spectral discretisation in space together with an Adams-Bashforth timestepping scheme. The timestepping routine itself will only find stable solution branches but will also allow solutions other than travelling waves to be found.

The nonlinear equations introduce three new parameters to the problem. These are $\sigma, \kappa$, and $\mu$ that represent the efficiency of the $\alpha$-, $\omega-$ and $\eta$ - quenching in the model respectively. The increase in the number of parameters in the problem means that an extensive search through parameter space is not feasible. However, the linear theory discussed earlier indicated that the results would not be too sensitive to particular selections in parameters that modify the profiles for the shear, $\alpha$-effect and diffusivity. It therefore seems more sensible to explore the effect of the quenching parameters in the nonlinear regime, and to keep the 'profile parameters' fixed at $E=10, z_{2}=0$ (so the profiles are as in Fig. 2). The boundary conditions are also held fixed.

\subsection{1. $x$ - and w-quenching}

The simplest extension to the nonlinear regime for the travelling wave solutions is to introduce $\alpha$-quenching as an equilibration mechanism for the field (i.e., set $\sigma=1$ without loss of generality $\mu=\kappa=0$ ). The branch of travelling waves is followed from the initial Hopf bifurcation at $D=-42.0$, as the magnitude of the order parameter $D$ is increased. The branch is shown in Figure 5 where the generated magnetic field is a monotonically increasing function of the dynamo number $D$; this is a feature of all the nonlinear solutions found in this section. The travelling waves remain stable for all the values of $D$ investigated, which is also a result that holds for all further choices of parameters. The lack of subsequent bifurcations to more complicated time-dependent behaviour means that the effects of varying the parameters (and hence the nonlinearities) on the spatial structure and the wavespeed can be investigated with relative ease in this system. Clearly a different type of nonlinearity (other than simple quenching) is needed to produce more complicated time-dependence in this system.

In Figure 5(b) the wavespeed $c$ is plotted as a function of dynamo number. It can be seen that the wavespeed decreases slightly as $-D$ 

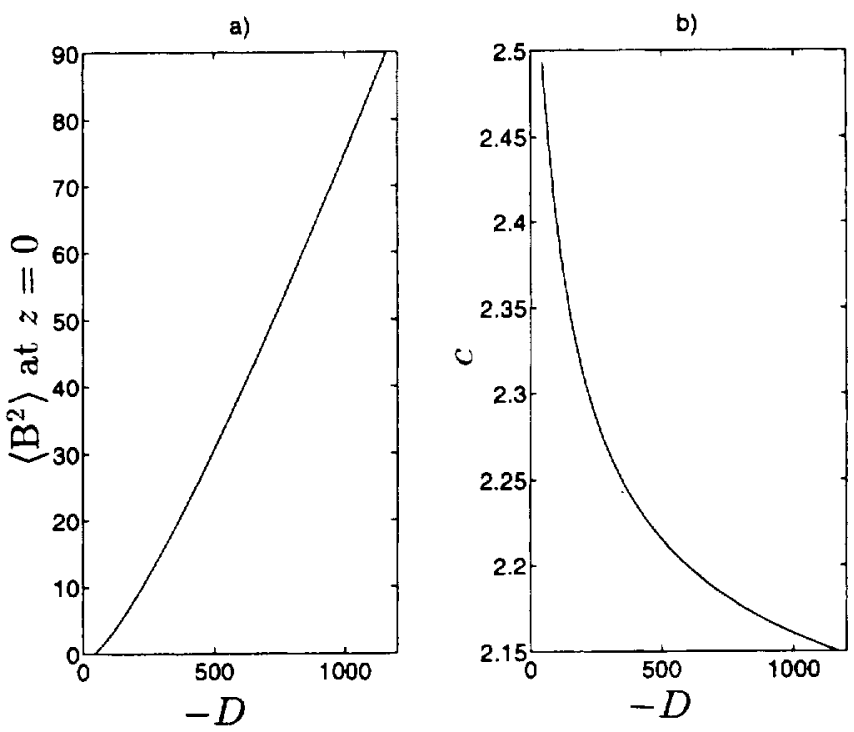

FIGURE $5 \alpha$-quenching - nonlinear solution: (a) Average magnetic energy $\left\langle B^{2}\right\rangle$ at $z=0$ versus dynamo number, $-D$ for the case $\sigma=1, \kappa=\mu=0$. (b) Wavespeed $c^{\prime}$ versus dynamo number with parameters as in (a). The wavespeed stays nearly constant (although a slight drop is noticeable).

increases, so the waves slow down as $|D|$ is increased; at first sight this seems a strange result. The quenching mechanism acts so as to reduce the effective value of the dynamo number until $D_{\mathrm{efr}} \sim D_{c}$. The wavespeed should therefore remain approximately constant (as noted in Noyes et al., 1984). The slight decrease in wavespeed is due to the modification of the profile for the $\alpha$-effect by the local action of the magnetic field, and can be understood in the context of the results of the linear theory discussed earlier. Figure 6 shows how the growth of magnetic energy in the area of overlap of the regions of $\alpha$-effect and shear quenches the $\alpha$-effect in that region. The $\alpha$-effect is now confined to a thin region near the top of the layer, and there is no longer a large region of overlap. Hence the dynamo is less efficient (as discussed earlier) and the wavespeed is less than that for the critical value of the dynamo number. The nonlinear toroidal and poloidal fields have a similar spatial dependence as the eigenfunction of the linear problem, as shown in Figure (6a). This indicates that the quenching acts simply 
(a)

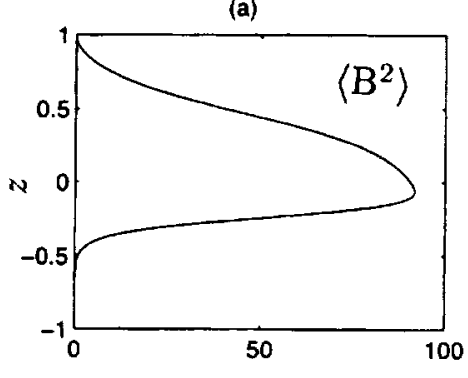

(b)

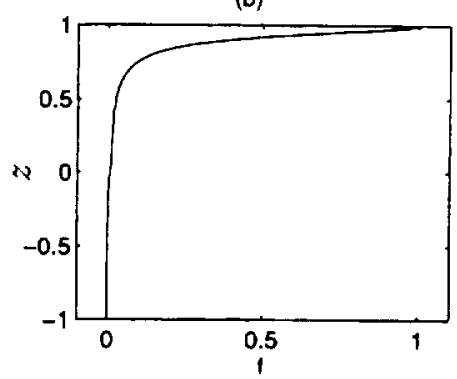

FIGURE 6 Nonlinear solutions ( $\alpha$-quenching) - $z$-dependence: (a) Graph of $\left\langle B^{2}\right\rangle$ (horizontal) versus $z$ (vertical) for $D=-1164$, other parameters as in Figure 5. The toroidal field is concentrated at and just below $z=0$ as in the linear eigenfunction. (b) Graph of $\alpha$-effect (horizontal) versus z (vertical). The $\alpha$-effect has been quenched to zero by the magnetic field throughout the bulk of the region, only reaching its unquenched level at $z=1$ where the toroidal field is set to 0

as an equilibration mechanism for the field, and does not change the spatial dependence of the solutions.

The case where $\kappa=1$ and $\mu=\sigma=0$ is now considered, so that only $\omega$ quenching is operating. Figure 7 shows that again the magnetic energy at $z=0$ is a monotonic function of $D$ and the travelling wave solutions remain stable. Moreover the wavespread of solutions does not vary significantly as $D$ is increased. In this case only a slight increase in wavespeed is observed for large dynamo numbers. The dynamo is therefore slightly more efficient for larger forcing. The solution for large dynamo numbers has a similar $z$-dependence to the eigenfunction of the linear theory, as in the case where $\alpha$-quenching is considered in isolation.

\subsection{2. $\eta$-quenching}

So far the nonlinear quenching has acted solely as an equilibration mechanism but it is now necessary to include the effect of the Lorentz force on the diffusion properties of the turbulence. Now we set $\mu=1$, so that $\eta$-quenching is in operation, ( $\alpha$-quenching also remains in operation as $\sigma=1$ ) and it is expected that the $z$-dependence of the solutions will change as the turbulent diffusion is modified by the action of the magnetic field. 

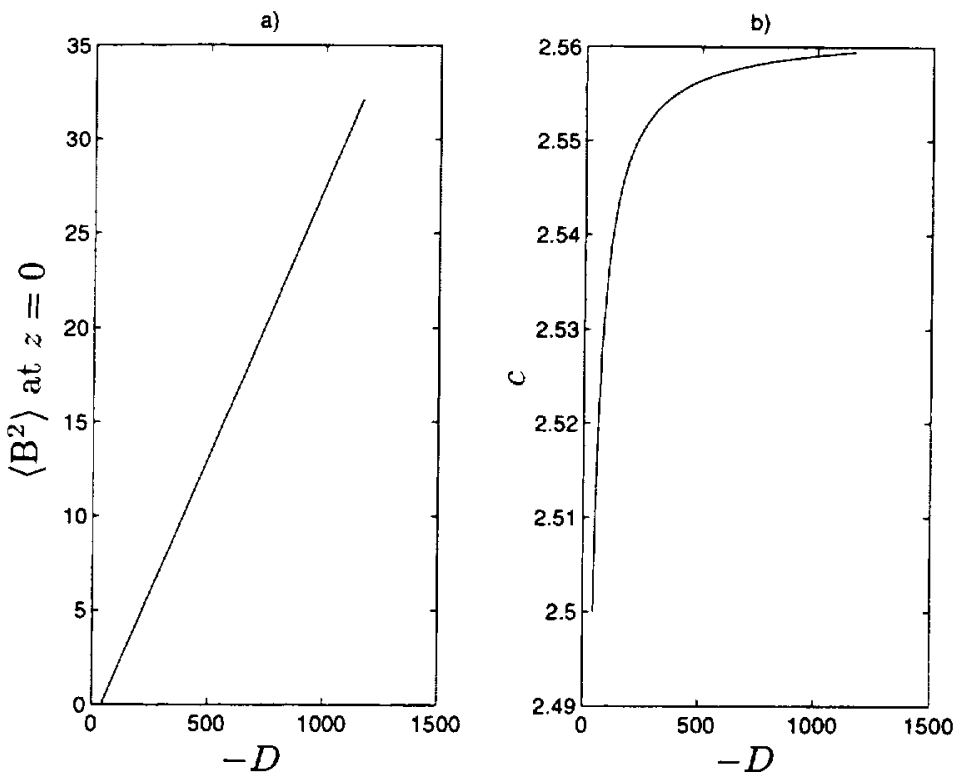

FIGURE 7 w-quenching nonlinear solutions: (a) As for Figure 5(a), but parameters are now $\kappa=1, \sigma=\mu=0$. (b) As for Figure 5(b), but parameters are as in (a). Again the wavespeed $c$ is largely unaffected ( $\sim 25 \%$ change), although a slight increase in $c$ may be detected.

In Figure 8(a) the dependence of the magnetic energy on dynamo number is displayed and when this is compared with Figure 5(a) it is clear that the toroidal field strength (and hence magnetic energy) that may be created is significantly larger for the case when $\eta$-quenching is included. This effect can be explained using either mathematical or physical arguments. It is necessary to understand how the reduction of the local value of the turbulent diffusivity can lead to an increase in the strength of the magnetic field that may be generated by dynamo action. The mathematical argument is simply that the reduction of diffusion locally by $\eta$-quenching leads to an increase in the value of the effective local dynamo number $D_{\mathrm{loc}}$. This follows immediately from the definition

$$
D_{\mathrm{loc}} \equiv D \frac{g\left(z, \overline{B^{2}}\right) f\left(z, \overline{B^{2}}\right)}{h^{2}\left(z, \bar{B}^{2}\right)}
$$



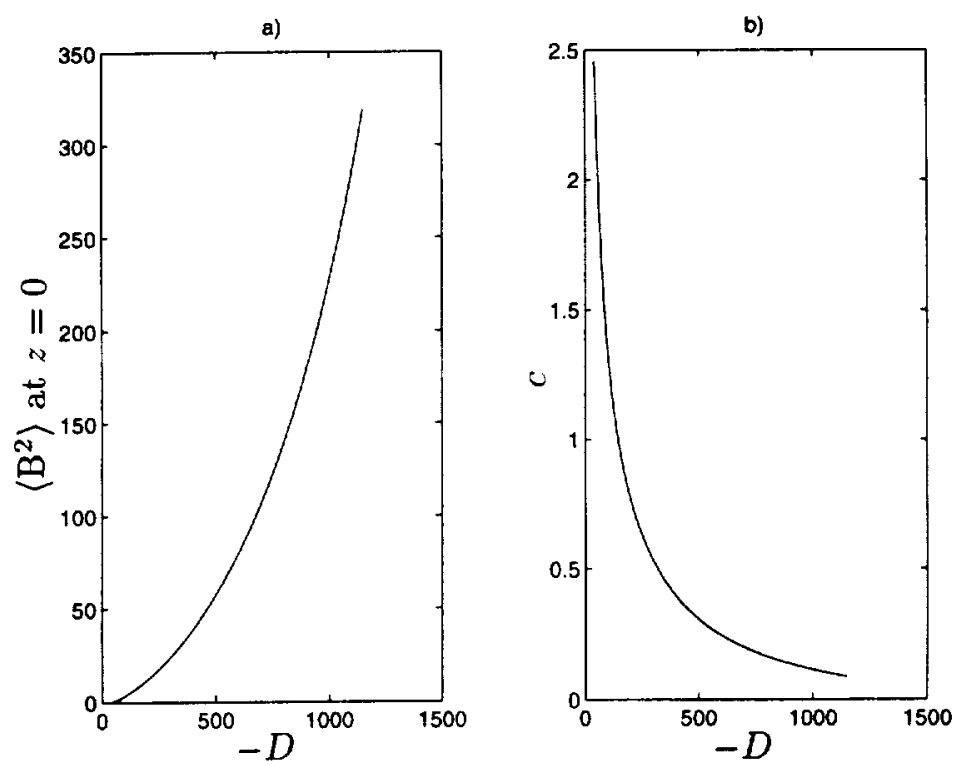

FIGURE $8 \quad \eta$-quenching (together with $\alpha$ and $\omega$-quenching): (a) As for Figure 5(a), but for parameter values $\sigma=\kappa=\mu=1$. The magnetic energy is much larger for a given dynamo number than for the case with just $\alpha$ and $\omega$-quenching. (b) As for Figure 5(b), but with parameters in (a). THe wavespeed is reduced to nearly zero as the dynamo number is increased. This is a new effect introduced by the $\eta$-quenching.

and assuming that $\alpha$ - and $\omega$-quenching are kept at the same level. An increase in $D_{\text {loc }}$ means that the local generation of field is more efficient for a given global dynamo number and this leads naturally to stronger fields. Physically, local dissipation via the turbulent convection is reduced by the action of the Lorentz force and this leads to the build up of magnetic energy locally and to significantly larger field strengths. At first there may appear to be a contradiction here, reducing dissipation leads to the build-up of stronger fields yet earlier it was argued that magnetic dissipation is vital to the Parker dynamo. How may the two be reconciled? The answer is that as the turbulent diffusion is quenched the form of the solution changes (see below) so that the regions of poloidal and toroidal field overlap. Diffusion is now not so essential to the operation of the dynamo as in the linear model because both the toroidal and poloidal fields are in the same location.

The most striking effect of introducing $\eta$-quenching can be seen in Figure $8(b)$ where the wavespeed is plotted as a function of dynamo 
number. In contrast to the cases where only $\alpha$ - and $\omega$-quenching were considered the wavespeed does depend significantly on the dynamo number. The wavespeed is a monotonically decreasing function of dynamo number, showing a sharp drop initially and appearing to tend to zero as $D \rightarrow \infty$. This drastic reduction in wavespeed is due to the fact that the turbulent diffusivity has been almost completely quenched by the magnetic field and molecular diffusion is the only mechanism for the transport of magnetic flux. This makes the dynamo significantly less efficient as diffusion of poloidal field from the convection zone to the overshoot region and of toroidal field in the opposite direction are both suppressed. As the dynamo is less efficient the wavespeed $c$ is decreased and the cycle period is increased for higher dynamo numbers. This result raises the question of how much the diffusivity may be quenched in astrophysical bodies-if the quenching is too strong the dynamo may cease to be efficient.

Another indication that the quenching is no longer acting simply as an equilibration mechanism comes from the spatial dependence of the solution as shown in Figure 9. The $z$-dependence of the $\eta$-quenched solution (particularly of the toroidal field) is very different from the eigensolution. This is because there is no longer a sharp change in the diffusivity in the region where the magnetic field is generated, so the rapid change in preferred lengthscales that occurs at $z=0$ in the linear theory (and in the nonlinear $\alpha$ - and $\omega$-quenching models) is no longer present. As a result the field is no longer confined near to $z=0$ and can penetrate further in to the region $z<0$, where the shear is strongest and so toroidal field may be easily produced. The slanted appearance of the toroidal field is due to the action of the shear and the time taken for the toroidal field produced by the shear to diffuse upwards into the region $z>0$. All these results demonstrate that the $\eta$ quenching mechanism has a very different effect on the nature of the solutions from the other types of quenching. It changes the form of the solutions and has a drastic effect on the wavespeed.

\subsection{Local Quenching}

The form of the (averaged) quenching selected in (15) is a simplification consistent with the hypothesis that the solutions are local and therefore represent the magnetic behaviour at a fixed latitude. The 

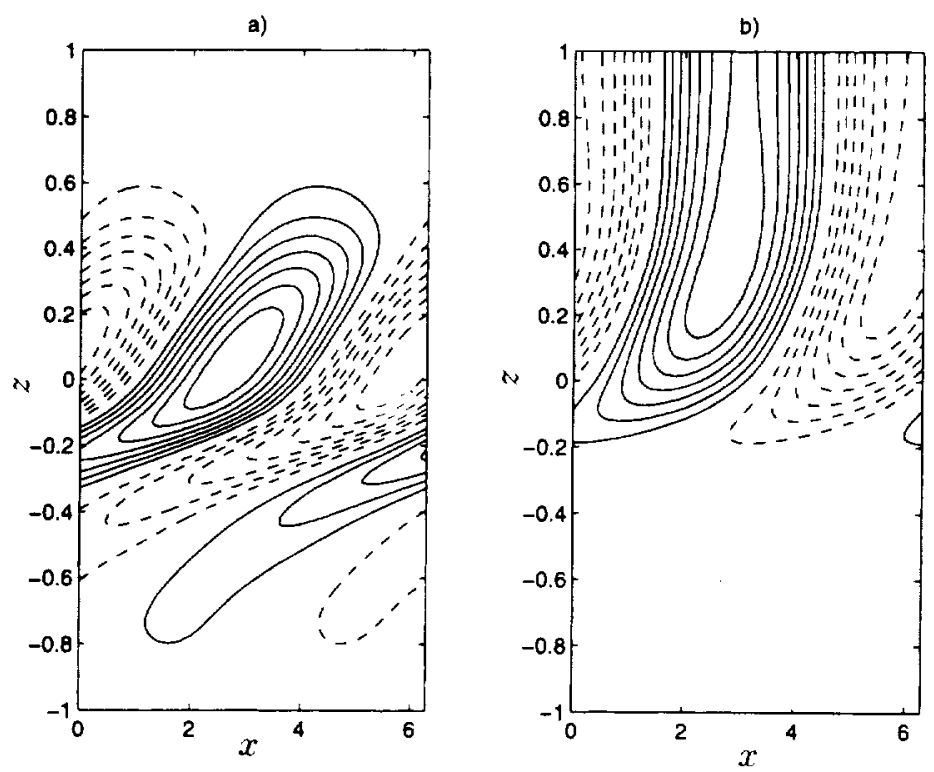

FIGURE $9 \quad \eta$-quenching-contour plots: (a) One wavelength of the toroidal field $B$, for the parameters in Figure 8 and $D=-1150$. The toroidal field may now penetrate into the region $z<0$. (b) One wavelength of $A$, for the parameters as above.

simplification to a one-dimensional ( $z$-dependent) form for the quenching enabled the nonlinear solutions to be followed using a continuation method and the radial dependence to be studied in detail. However it is important to check that the main conclusions remain valid if the form of the quenching is changes to a 'local' (fully two-dimensional) form. Hence the calculations are repeated for the case where the quenching is local and the definitions in (15) are replaced by

$$
Q_{\sigma}=\frac{1}{1+\sigma B^{2}(x, z)}, \quad Q_{\kappa}=\frac{1}{1+\kappa B^{2}(x, z)}, \quad Q_{\mu}=\frac{1}{a+\mu B^{2}(x, z)} .
$$

The equation for the velocity perturbations is again decoupled by setting $\Upsilon=0$. The equations are now fully two-dimensional and are therefore too complicated to solve using a continuation method. Stable solutions are found using the time-stepping code described earlier. 
The results of the integrations show that the solutions behave in an analogous manner to those for the case with averaged quenching. For example for simple local $\alpha$-quenching only, we set $\sigma=1, \kappa=\mu=0$. For this choice of parameter values the travelling waves remain stable as $D$ is increased into the highly nonlinear regime. As in the case where the quenching is proportional to the average magnetic energy of the wave, the magnetic field strength is a monotonic function of dynamo number and the speed of travel of the waves varies only marginally. For all other calculations where the velocity equation is decoupled and local quenching mechanisms are the sole nonlinearity, travelling waves remained stable. Hence the introduction of local dependence to the quenching models has very little effect on the nature of the solutions. As in the case of global quenching the temporal behaviour of solutions remains uncomplicated.

Quenching mechanisms are particularly simplified parametrisations of the effect of the Lorentz force, being local in space and instantaneous in time. This inherent simplicity means that the form of the solutions is not sensitive to the exact choice of the nature of the mechanism-i.e. whether the mechanism is averaged (one-dimensional) or local (two-dimensional). In Section 6 it will be demonstrated that this is not the case for the more-complicated (and realistic) Malkus-Proctor nonlinearity, where the particular form of the interaction can have large consequences for the nature of the solutions.

\subsection{Summary of Quenching Results}

In the above sections a thorough investigation of the nonlinear behaviour of the travelling waves has been conducted. The important results are summarised below. The travelling waves remain stable for all choices of parameters and all values of the dynamo number investigated. This indicates that the quenching mechanisms (whether local or averaged) considered do not naturally lead to modulation of the waves. It may be that a more realistic formulation of the problem where the Lorentz force is allowed to act back directly on the velocity will lead to more complicated time-dependence and this is investigated in Section 6.

$\alpha$ - and $\omega$-quenching have similar properties. Both act purely as equilibration mechanisms. They have little effect on the spatial 
structure of the solutions. Also the speed of the travelling waves remains roughly constant as the dynamo number is increased. However, the introduction of $\eta$-quenching changes both the spatial structure of the waves and also the wavespeed. The wavespeed is reduced as the dynamo number (and hence magnetic energy of solutions) is increased. The dynamo becomes less efficient as the turbulent diffusion is reduced by the strong fields. The initial Hopf bifurcation to travelling waves may be subcritical if the $\eta$-quenching is allowed to be significantly stronger than the $w$-quenching.

\section{THE MALKUS-PROCOTOR NONLINEARITY - ROUTES TO CHAOS}

In this section I will demonstrate that it is only by considering a sufficiently realistic (and therefore complicated) nonlinearity that a model can be constructed that exhibits transitions to complicated temporal behaviour. It is therefore vital to include the rôle of the Lorentz force in generating velocity perturbation via the MalkusProctor effect. These results will prove to be the most interesting and important when describing the modulation of the basic solar cycle.

We therefore consider the fully-coupled nonlinear system given by (6). The boundary conditions that are imposed on the magnetically driven velocity $v$ must be consistent with the (implied) conditions satisfied by the shear $g$. In this case, it is logical to choose periodic boundary conditions in the $x$-direction and

$$
v(x,-1)=0, \quad \frac{\partial v}{\partial z}(x, 1)=0
$$

as the conditions on the top and bottom of the box.

\subsection{Global (Averaged) Interaction}

\subsection{Derivation of Equations}

The travelling wave models discussed so far are local models in the sense that they contain no latitudinal dependence of the parameters. In 
this case it is also reasonable to adopt nonlinearities that are averaged over the period of a wave in the $x$-direction, as the primary interest is in the radial (z) dependence of solutions and their temporal behaviour. Calculations that include local quenching mechanisms have been considered, and local interaction via the Malkus-Proctor effect will be considered later in this section. For now, the $\alpha$-effect, shear and turbulent diffusion remain quenched with the average magnetic field energy so that $f\left(z, \bar{B}^{2}\right), g\left(z, \bar{B}^{2}\right)$ and $h\left(z, \bar{B}^{2}\right)$ remain as defined in $(8)-(10)$. If the quenching is averaged in the latitudinal direction then for consistency the perturbation to the velocity should only arise due to the Lorentz Force averaged over the period of the travelling wave in the $x$-direction. In this case (6) becomes

$$
\begin{aligned}
& \frac{\partial A}{\partial t}=h\left[\frac{\partial^{2} A}{\partial x^{2}}+\frac{\partial^{2} A}{\partial z^{2}}\right]+f B \\
& \frac{\partial B}{\partial t}=h\left[\frac{\partial^{2} B}{\partial x^{2}}+\frac{\partial^{2} B}{\partial z^{2}}\right]+\frac{\partial h}{\partial z} \frac{\partial B}{\partial z}+D\left[g(z)+\frac{d \bar{v}}{d z}\right] \frac{\partial A}{\partial x} \\
& \frac{\partial \bar{v}}{\partial t}=\tau h\left[\frac{\partial^{2} \bar{v}}{\partial x^{2}}+\frac{\partial^{2} \bar{v}}{z^{2}}\right]+\tau \frac{\partial h}{\partial z} \frac{\partial \bar{v}}{\partial z}+\operatorname{sign}(D)\left[\frac{\partial B}{\partial z} \frac{\partial A}{\partial x}-\frac{\partial A}{\partial z} \frac{\partial B}{\partial x}\right]
\end{aligned}
$$

where an overbar indicates a horizontal average over the period of a wave in the $x$-direction. These equations with the averaged effects of the Lorentz Force form a starting point for this investigation. Travelling wave solutions can be found by expanding the poloidal and toroidal fields in a Fourier expansion moving with the wavespeed as in (16). In a similar way ordinary differential equations for the coefficients $a_{j}^{s}, a_{j}^{c}, b_{j}^{s}$ and $b_{j}^{s}$ can be derived, though this time they are coupled to an equation for $\bar{v}(z)$. The equations become

$$
\begin{aligned}
h\left[b_{j}^{\prime \prime c}(z)-j^{2} b_{j}^{c}(z)\right]+c j b_{j}^{s}(z)+D\left(g+\frac{d \bar{v}}{d z}\right) j a_{j}^{s}(z)+h^{\prime} b_{j}^{\prime c}(z) & =0 \\
h\left[b_{j}^{\prime \prime s}(z)-j^{2} b_{j}^{s}(z)\right]-c j b_{j}^{c}(z)-D\left(g+\frac{d \bar{v}}{d z}\right) j a_{j}^{c}(z)+h^{\prime} b_{j}^{\prime c} & =0, \\
h\left[a_{j}^{\prime \prime}(z)-j^{2} a_{j}^{c}(z)\right]+c j a_{j}^{s}(z)+f b_{j}^{c}(z) & =0 \\
h\left[a_{j}^{\prime \prime s}(z)-j^{2} a_{j}^{s}(z)\right]-c j a_{j}^{c}(z)+f b_{j}^{s}(z) & =0,
\end{aligned}
$$


for $j=1, m$, together with

$$
\tau h \frac{d^{2} \bar{v}}{d z^{2}}+\tau h^{\prime} \frac{d \bar{v}}{d z}+\operatorname{sign}(D) \frac{1}{2} \sum_{j=1}^{m} j\left[b_{j}^{\prime} a_{j}^{s}-b_{j}^{\prime} a_{j}^{c}-b_{j}^{s} a_{j}^{\prime}+b_{j}^{c} a_{j}^{\prime s}\right]=0 .
$$

These equations (23)-(24), together with the appropriate boundary conditions on the toroidal and poloidal field defined in (12) and

$$
\bar{v}(-1)=\frac{d \bar{v}}{d z}(1)=0
$$

constitute a two point boundary value problem. The stability of these travelling wave solutions may be determined using the pseudospectral/ finite difference timestepping scheme described earlier. Indeed, as bifurcations to more elaborate time-dependent behaviour are expected, this timestepping scheme will be useful in locating stable quasiperiodic and chaotic solutions. Here the properties of these travelling waves are investigated in the expectation that they will lead to some understanding of the Malkus-Proctor effect that may prove useful in analyzing the full problem.

\subsubsection{Properties of Travelling Waves: Wavespeed and Shear Profiles}

Rather surprisingly (and perhaps disappointingly), the travelling waves remain stable for all the combinations of parameter values selected. To obtain the required modulation therefore, one must consider the full problem, with local interaction of the magnetic and the velocity fields, defined by (6) (as in Section 6.2). The travelling waves do however show different behaviour than those found for simple quenching mechanisms. Initially the Malkus-Proctor effect is set to be the only nonlinearity in the problem and $\kappa, \sigma$ and $\mu$ are all set to be zero and the magnetic Prandtl number $\tau$ is set to be unity. All other parameters relating to the shape of the profiles for the $\alpha$-effect, shear and turbulent viscosity are set to be the same as in Figure 2.

Here (as before) negative dynamo numbers are studied. Figure 10 demonstrates that both the magnetic energy and wavespeed are increasing functions of the magnitude of the dynamo number. The wavespeed increases by a significant amount. The $z$-dependence 

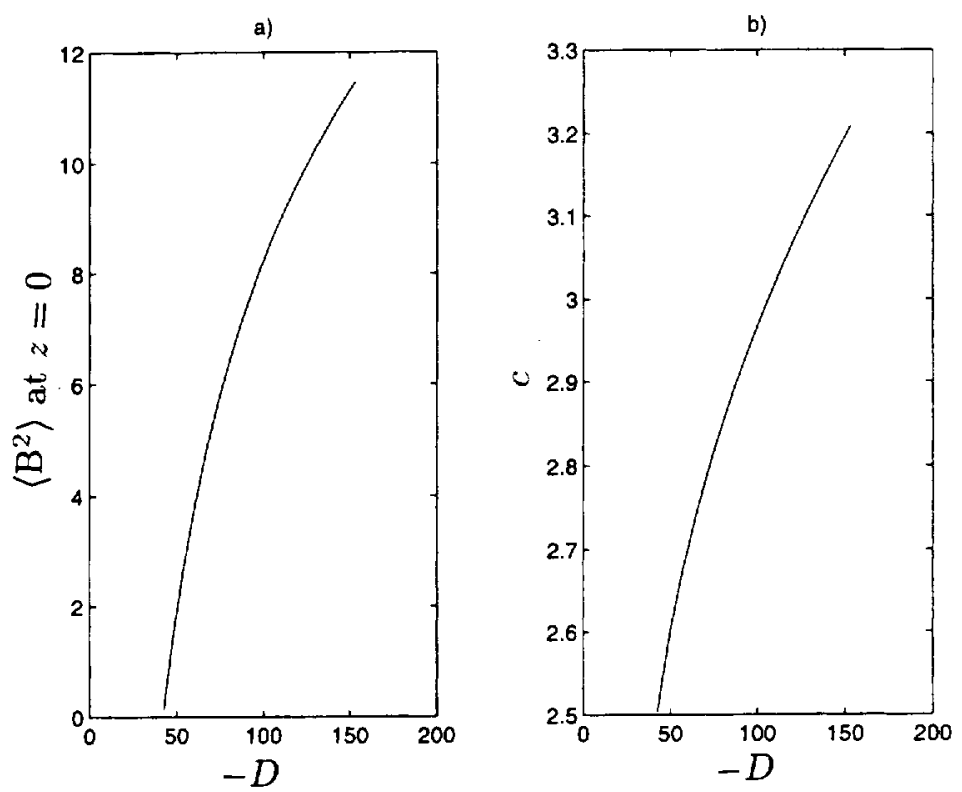

FIGURE 10 Malkus-Proctor only - nonlinear solutions: (a) Average magnetic energy $\left\langle B^{2}\right\rangle$ at $z=0$ versus dynamo number, $-D$ for the $\sigma=\kappa=\mu=0, \tau=1$. (b) Wavespeed $c$ versus dynamo number with parameters as in (a). The wavespeed increases as the magnitude of the $D$ is increased.

of the toroidal field in the nonlinear regime is similar to that for the eigenfunction with the only change being that the Lorentz Force generates a shear lower down (i.e. at smaller values of $z$ ) than the region where the magnetic energy is concentrated. This shows one important difference between quenching mechanisms and the MalkusProctor effect-whilst quenching mechanisms may only act where the field is strong, the Lorentz force may drive shears in places with weak(er) fields. This is because the Lorentz Force term in the velocity equation depends on gradients of the field (and not soley on the absolute magnitude). If the other nonlinear effects are kept at zero, then changing the value of $\tau$ in the averaged velocity equation has no effect on the nature of the solutions. Indeed it may be removed from the equations by rescaling the magnetic field. Hence if $\sigma=\kappa=\mu=0$ then $\tau=1$ without loss of generality. 
Having understood the case with the Malkus-Proctor effect only, we now wish to include the quenching mechanisms to see what effect (if any) they may have. A large number of combinations of parameter values are available to us, and here only a small subset is presented. The first quenching mechanism to be added is $\alpha$-quenching The magnetic energy is less if both nonlinearities are included together than if the nonlinearities are considered in isolation (unsurprisingly). The dependence of the wavespeed is slightly more complicated; initially if both mechanisms are included the wavespeed is higher as the MalkusProctor mechanism tends to increase the wavespeed. However if $D$ is increased further the speed drops below that of the case where the $\alpha$ quenching is acting in isolation which demonstrates that the two mechanisms must interact in a more complicated manner than simply adding the two effects together.

If $\omega$-quenching and $\eta$-quenching are added the story is much the same - the magnetic energy is less if the Malkus-Proctor effect is included. For the wavespeed, the $\eta$-quenching is again the dominant nonlinearity, slowing the wave down for the reasons discussed in earlier sections - the Malkus-Proctor effect does have some effect in counteracting this behaviour, but it is clear that the efficiency of the dynamo is diminished by the suppression of the turbulent diffusion.

\subsection{Local Interactions}

The global interaction described in the previous secion failed to lead to the complicated time-dependence anticipated. For this reason we return to the full problem where the interactions of magnetic fields with velocity fields are local, and no averaging takes place. If the Lorentz force drives a large-scale velocity locally then for selfconsistency the quenching mechanisms that parametrise the effects of the Lorentz Force on the small-scale turbulence should also depend on the local values of the magnetic fields strength. Hence the definitions of $f, g$ and $h$ representing the $\alpha$-effect, shear and turbulent diffusion are as in the local models described in equation (20).

The local quenching makes it very difficult to use continuation methods on the problem as the problem is now fully two-dimensional. For this reason the timestepping routine is used to find stable solutions for this problem. 


\subsubsection{Malkus-Proctor Effect Alone}

As in the last section, the quenching mechanisms are initially switched off by setting $\sigma=\kappa=\mu=0$ and the Prandtl number $\tau$ is set to be unity. For $|D|<D_{\text {crit }}=42$ both the magnetic field and velocity field decay away as predicted by linear theory but as the dynamo number is increased past this value dynamo action sets in at a supercritical Hopf bifurcation to travelling waves. For $D$ just greater than this critical value stable travelling waves are found - the solution is periodic, but is constant in a frame of reference travelling with the wavespeed. Analysis of the solutions is easier if this basic oscillation associated with the speed of travel of the wave is removed, which can be accomplished by examining some global properties of the solutions. The average magnetic energy $E_{B}$ and the energy kinetic energy of the magnetically driven velocity field $E_{K}$ are calculated, where

$$
E_{B}=\frac{1}{V} \int_{V} B^{2} d x d z, \quad E_{K}=\frac{1}{V} \int_{V} v^{2} d x d z
$$

where $V$ is the domain of integration $(0<x<2 \pi,-1<z<1)$.

For travelling wave solutions we except $E_{B} \rightarrow$ const. and $E_{K} \rightarrow$ const. and the trajectory to tend to a fixed point in the $\left(E_{B}, E_{K}\right)$ phase plane. For a weakly nonlinear solution both $E_{B}$ and $E_{K}$ are reasonably small.

The solutions for the toroidal and poloidal field are shown in Figure 11 - these are very similar to the eigenfunctions, which is not surprising as the induced velocity $v$ is very small and so will have only a weak effect on magnetic field generation. The figure shows the wave travelling along the interface from left to right; nearly a complete cycle of the wave, which has a period $T \sim 2.5$, is displayed. The wavespeed can therefore be calculated as $c \sim 2.5$ ( $1 \mathrm{~d}$.p.) which is consistent with the linear theory.

The magnetically-driven velocity perturbations are displayed in Figure 12. As the velocity is driven by the quadratic Lorentz force its $x$-dependence is different to that of the magnetic fields. In this case it is clear that $B \sim B_{0} \sin (x-c t)$ and so it is natural that the velocity $v \sim v_{0}+v_{2} \sin 2(x-c t)$. It is the resultant shear that is important in equilibrating the dynamo, and it can be inferred that this is concentrated at small values of $z$. (The presence of this shear can be 

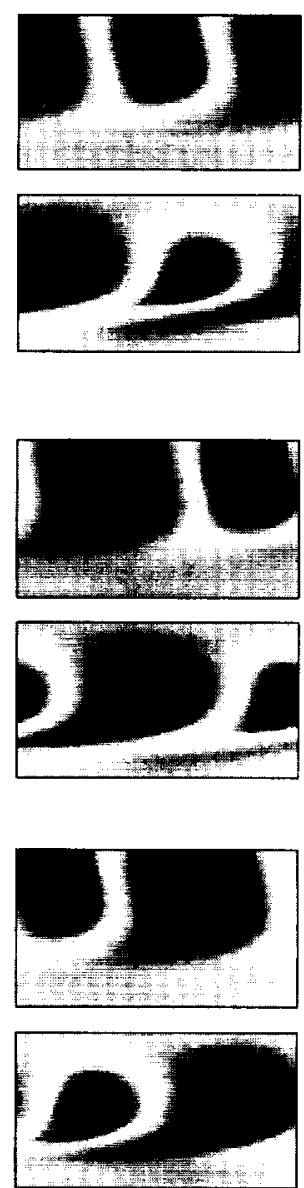
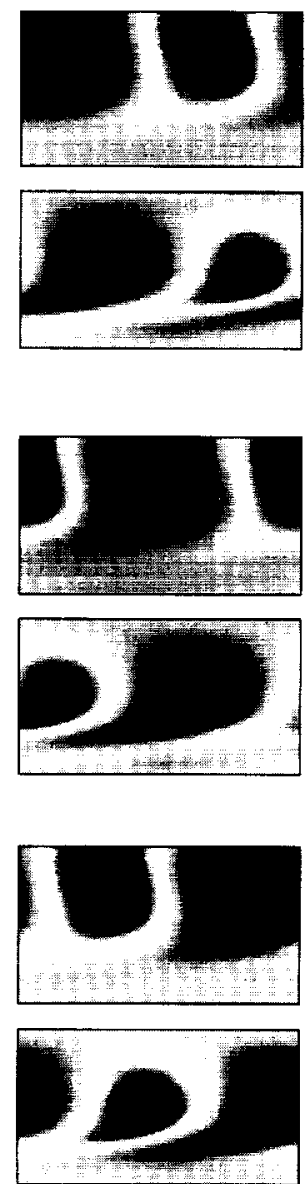

FiguRE II Magnetic field Travelling Waves. The figure shows the form of the solutions for A (top) and B (bottom) as time progresses for $D=-50$. The sequence should be viewed from left to right and top to bottom. Each snapshot is separated by a lime $t=0.4992$. Strong positive fields are shown in red whilst strong negative fields are in bluc. (Sec Color Plate 1).

detected by the rapid change of colours from blue to red to green near $z=-0.75$.) In this respect this model is similar to the averaged model considered in the previous section as the driven shear appears at small values of z away from the region of maximum magentic field. This shows once again that the Malkus-Proctor effect is non-local and therefore is more sophisticated than simple quenching mechanisms. 

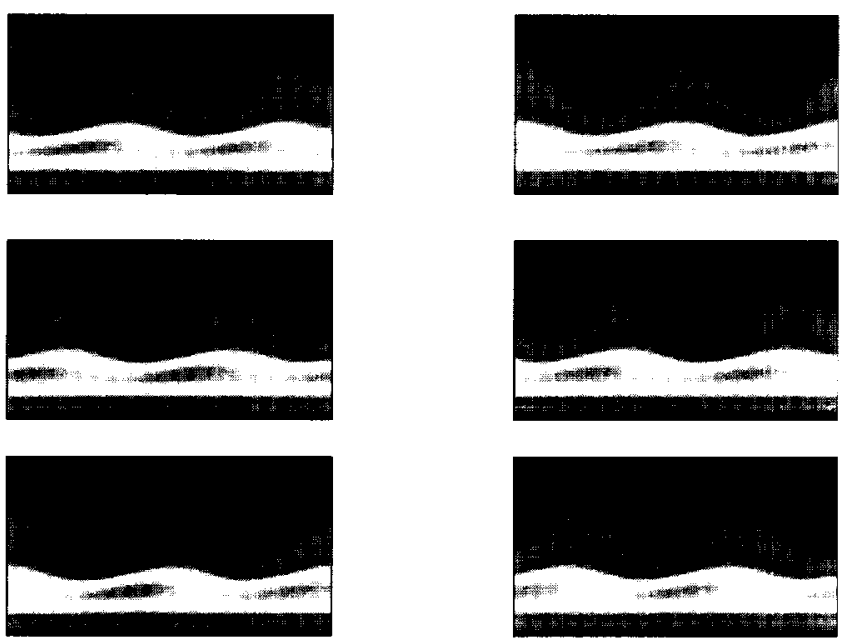

FIGURF 12 Velocity Perturbations Travelling Waves. The figure shows the form of the solutions for $r$ as time progresses for $D-50$. The velocity perturbations oscillate with half the period of the magnetic field. The snapshots correspond to those in Figure 11. The presence of a radial shear near $=-0.75 \mathrm{can}$ be inferred from the rapid colour change. (See Color Plate II).

The velocity perturbations migrate in the same direction as the magnetic field with the same wavespeed, but becaluse of the $x$ dependence of the perturbations, the period of the travelling waves is half that of the magnetic fields: i.e. $B\left(\alpha e^{i \omega t} \Rightarrow v k e^{2 i \omega t}\right.$.

If $|D|$ is increased further, the travelling waves grow in amplitude until they become unstable in a secondary Hopf bifurcation to modulated waves. For these modulated waves both the magnetic energy $E_{B}$ and the kinetic energy $E_{K}$ oscillate about a mean value as shown in Figure 13 with the period of oscillation being 0.46 . Hence for these parameter values, trajectories in energy space tend 10 a limit cycle as shown in Figure 13(c). This limit cycle corresponds to a twotorns when the basic oscillation due to the travelling waves is reintroduced.

The modulation is apparent in Figure 14 where the structure of the solution is now very different to that of the eigenfunction. After the secondary Hopf bifurcation the solutions not only travel but 'breathe' as they move along the interface. As well as this change in the 

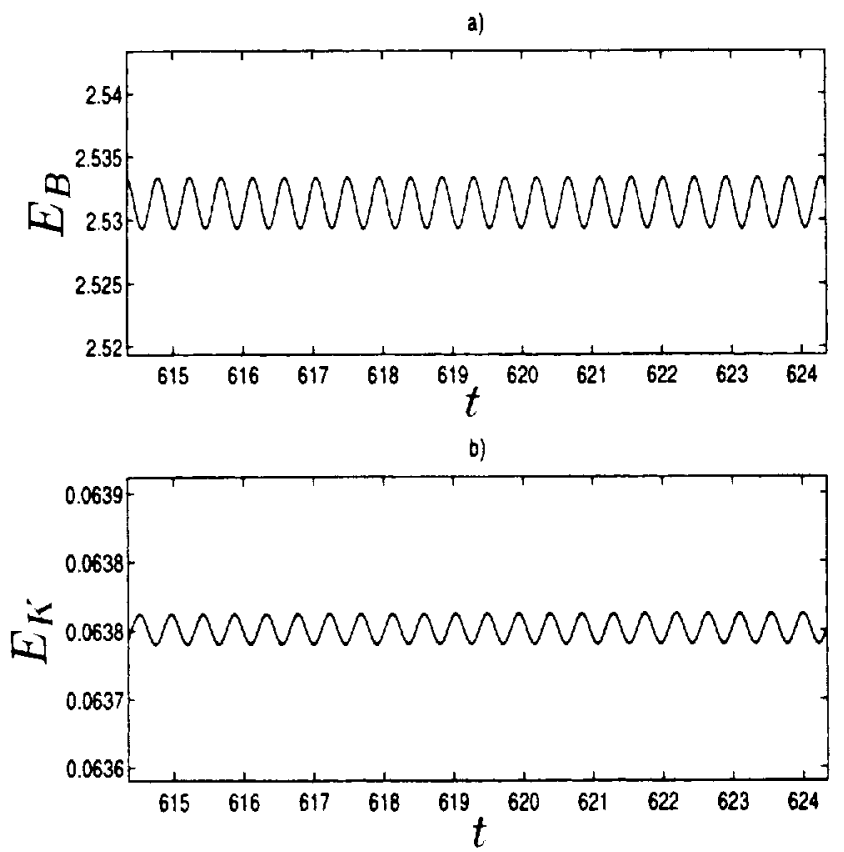

FIGURE 13 Modulated waves periodic energies. (a) Magnetic Energy $E_{B}$ for $D=$ -150 . (b) Kinetic Energy $E_{\mathrm{v}}$ for $D=-150$.

temporal behaviour of the solutions, the spatial dependence has clearly altered dramatically. The velocity field produced by the large-scale Lorentz force near $z=-1$ is now strong enough to generate some toroidal field. Two properties of this induced field are immediately apparent from Figure 14. The first is that this field varies on a smaller scale than the field in $z>-0.5-$ in this layer $B \sim \sin 2 x$. This can be attributed to the fact that this field is generated by the velocity perturbation and not the imposed shear and that it is entirely contained in a region where the diffusion is small and so the natural lengthscale is also smaller. The second property concerns the speed of propagation of the waves. The magnetic field in this layer travels very slowly to the right - a fact that can be seen by comparing the position of the strong field in the first and last frame in Figure 14. The speed of propagation of this field can be estimated to be $c \sim 2 \pi / 27=0.23$. The dynamo waves travel more slowly in this region because the diffusivity 

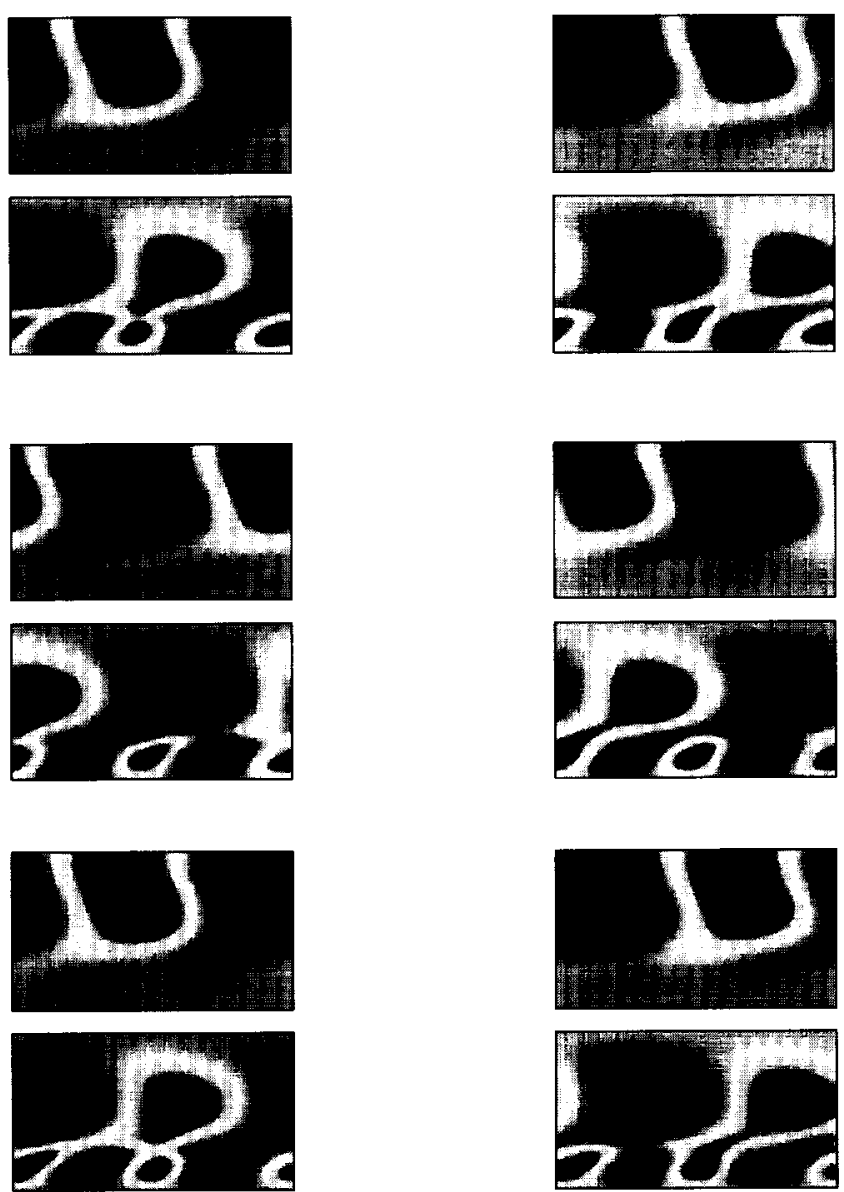

FIGURE 14 Magnetic Field Modulated Waves. As for Figure 11, but $D=-150$. The velocity field has acted back producing toroidal field at small values of $z$. This magnetic field has a smaller scale as it is produced by the velocity perturbations (which have a characteristic length-scale hall that of the main field) in a region with a smaller value of the diffusivity. It travels slowly to the right for the same reason. (See Color Plate III).

is small in this layer and the dynamo is therefore less efficient and hence slower. The magnetic field that is generated by the imposed shear for larger values of $z$ continues to travel relatively quickly and is largely unaffected by the magnetic field in the layer of reduced diffusivity. There is some interaction however, when a fast-moving 
region of strong positive field passes over a slow-moving region of positive field (as in the first snapshot on the left-hand side) these fields merge together and the radial (and latitudinal) gradients in the field will be small in the region. However, when strong positive field passes over strong negative field (as in the last snapshot on the right) large gradients in field are created. These are important in generating the velocity perturbations via the Malkus-Proctor effect.

The velocity perturbations corresponding to this magnetic field are shown in Figure 15. The snapshots show that the velocity at the top of the domain resembles that for the travelling wave case. At the bottom, however, the velocity has quite a different form: four nodes, which oscillate and travel slowly to the right, appear in this layer of reduced diffusion. The size and stregth of these nodes at any given time can be understood in terms of the relative positions of the fast-moving and slow-moving magnetic field. If, as explained earlier, the proximity of strong negative toroidal field and strong positive field leads to large gradients in the field, then these gradients are responsible for the generation of velocity perturbations.
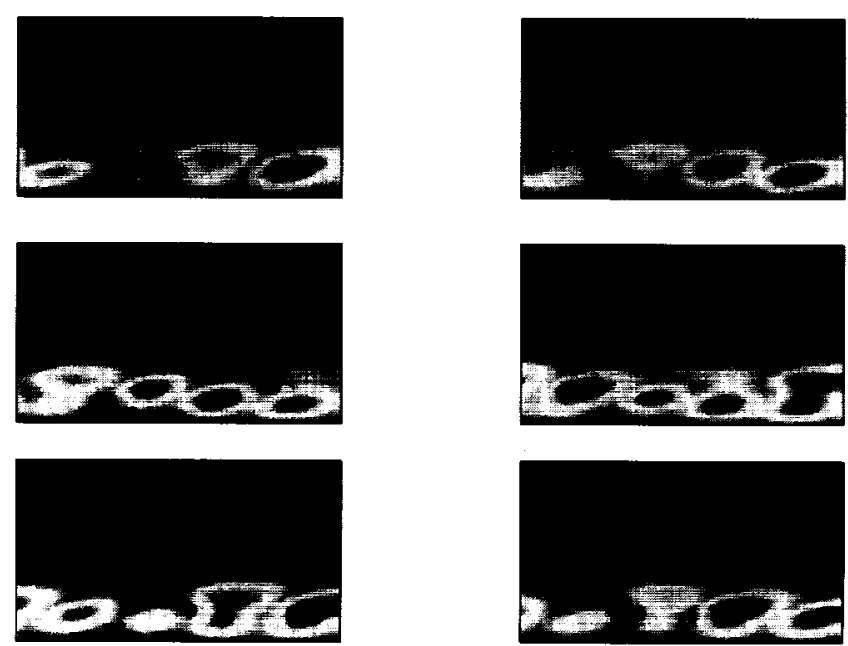

FIGURE 15 Velocity Perturbations Modulated Waves. The figure shows the form of the solutions for 1 is time progresses for $D=-150$. Velocity perturbations are drived by gradients in the mignetic tield. (See Color Plate IV). 
However this effect is not instantaneous - the velocity takes some time to respond to the magnetic field, and so there is a time-lag between the magnetic field and the generation of shear. It is well known from the work of Yoshimura (1975) that the introduction of a time-lag to the nonlinearities in the dynamo equations can lead easily to complicated time-dependence. The Malkus-Proctor effect, by including the equation for the large-scale velocity, introduces this time-lag in an entirely natural way. This is in contrast to the simple quenching mechanisms discussed previously, where the small-scale turbulent velocity field has an instantaneous response to the magnetic field which is local in space.

If the dynamo number is further increased, the modulated waves persist, with an increase in amplitude and frequency. However for $D=-330$ the nature of the solutions is different as shown in Figure 16 where the basic oscillation in the magnetic and kinetic energy is itself modulated on a longer timescale. This indicates that the solution has undergone a tertiary Hopf bifurcation where a third frequency of
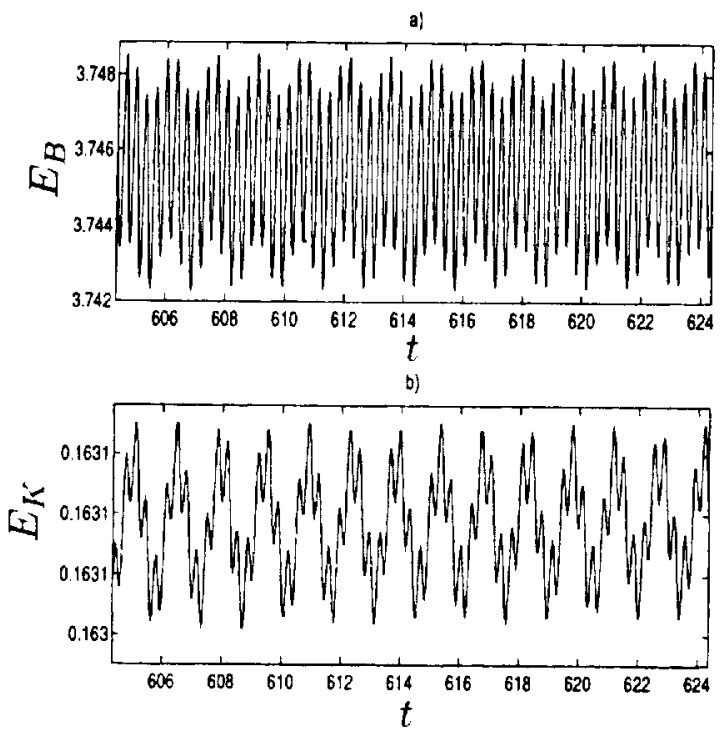

FIGURE 16 Quasiperiodic waves (a) As for Figure 13 (a), but $D=-330$. (b) As for Figure 13 (b), but $D=-330$. The energies are now quasiperiodic. 
oscillation has been introduced to the problem. Motion now lies on a two-torus in energy-space (corresponding to a three-torus in the extended system where the basic wavespeed is included). The spatialdependence for this type of solution is very similar to that for the modulated wave described earlier.

As $D$ is increased further this torus breaks down and the solutions become chaotic as shown in Figure 17. The 'ghost' of a torus still remains for this parameter value $(D=-400)$ as shown in Figure $17(\mathrm{c})$. In this case however the nature of the spatial dependence of solutions is somewhat different as shown in Figure 18. The solution is still characterised by two layers of interacting dynamo waves travelling at different speeds. The horizontal scale for the variation of the field is similar in both layers and the two layers interact substantially. This implies that the velocity perturbations are now as important as the imposed shear at the top of the box. If $D$ is increased further, chaotic solutions of ever-increasing complexity are found until problems with numerical accuracy prevent more interations at $D=-550$.

This sequence of calculations includes a variety of behaviour and therefore raises a number of questions about the processes involved here. The first question involves the bifurcation sequence. which is exactly that found in the low order model of Jones et al. (1985) and similar to that for the third-order system examined in Tobias et al. (1995). It is important to discover whether the bifurcations that the system undergoes are simply the result of a special choice of model parameters or if the sequence is robust. The model exhibits both interesting temporal and spatial behaviour. Are the two linked? That it, does the modulation follow simply from the interaction between the two layers of dynamo waves travelling at different speeds, or is the spatial behaviour a secondary effect of the modulation that simply enhances the behaviour already present?

\subsubsection{Solutions for Constant $\eta$}

The complicated two-layer structure for the toroidal field in the previous model is due to the disparity in the value of the diffusivity at the top and bottom of the domain. We may remove this structure by considering solutions to a model with constant diffusivity (i.e., a diffusion that is independent of both position and magnetic field 

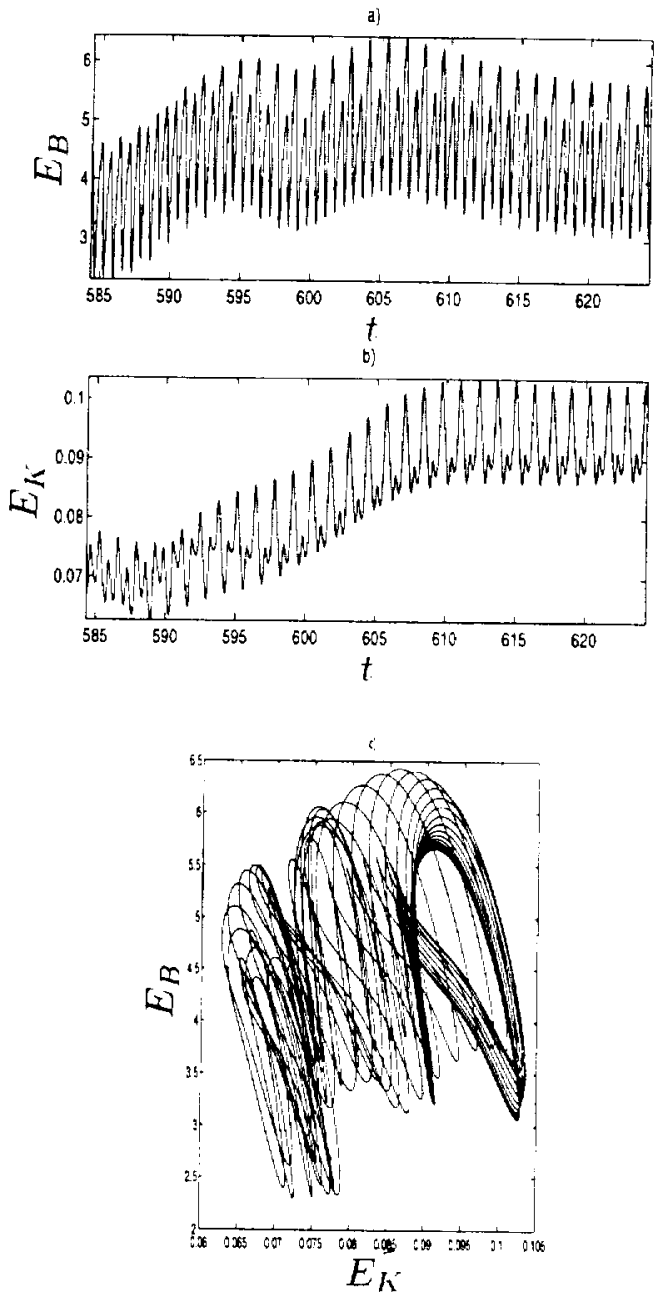

FIGURE 17 Chalotic solutions (a) As for Figure 13 (a), but $D=-400$, (b) As or Figure $13(\mathrm{~b})$, but $D=-400$. (c) (haotic attractor. The energies are now chatotically modulated.

strength). For this model, therefore $\hat{h}(z)=1$, and all other variables and parameters are as in the previous section.

Here dynamo action to travelling waves sets in at a supercritical Hopf bifurcation at $D=-83$. This value is greater than that for the variable diffusivity model because the diffusion is now $O(1)$ every- 

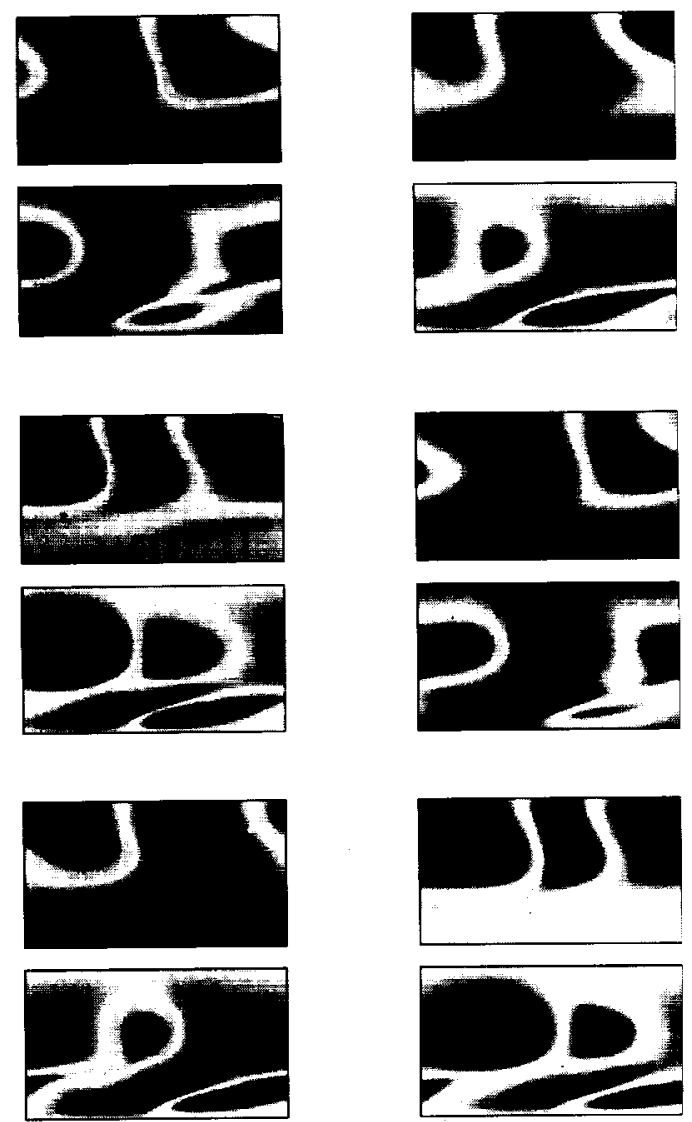

FIGURE 18 Magnetic Field Spatio-temporal Chaos. As for Figure 11 , but $D=-400$. (Sce Color Plate V)

where and so the shear and $\alpha$-effect must be larger to overcome dissipation. Again the travelling waves have constant magnetic and kinetic energy. Snapshots displaying the spatial dependence of the toroidal and poloidal field are shown in Figure 19, where it is apparent that the $z$-dependence of the solutions is less complicated than in the previous model. It is also interesting to note that the wavespeed of these solutions is $c \sim 2.8$, larger than for the marginally supercritical solutions for the previous model so the $\mathrm{O}(1)$ diffusion throughout the layer obviously increases the efficiency of the dynamo. This simple $z$-dependent structure is also found for the velocity perturbations. 

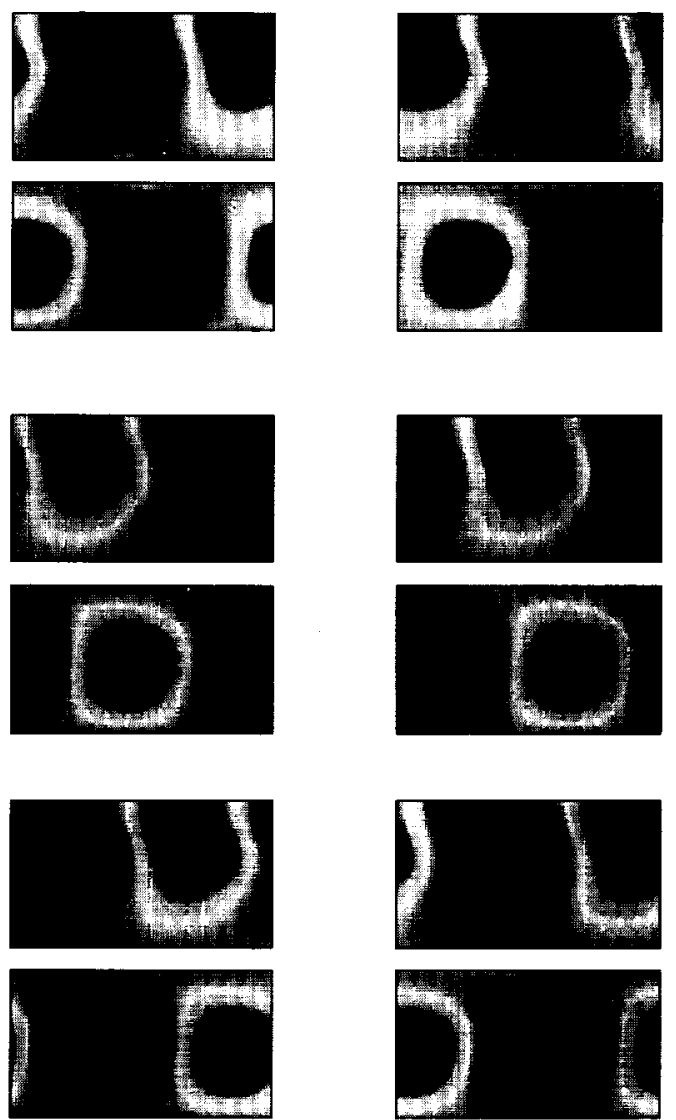

FIGURE 19 Magnetic Field - Travelling Waves. The figure shows the form of the solutions for $A$ (top) and $B$ (bottom) for the case where $\eta$ is a constant $D=-90$. (See Color Plate VI)

Increasing $|D|$ again leads to a secondary Hopf bifurcation and modulated waves where the magnetic and kinetic energy both vacillate. The spatial structure of the modulated waves remains simple as shown in Figure 20, with no hint of the complicated layer structure and interaction between waves travelling at different speeds that characterised the solutions for the model with variable diffusivity. The modulation is clearly visible as the relative strengths of the positive and negative fields oscillate as the wave propagates. This is particularly clear in the snapshots of the poloidal field - in the first 

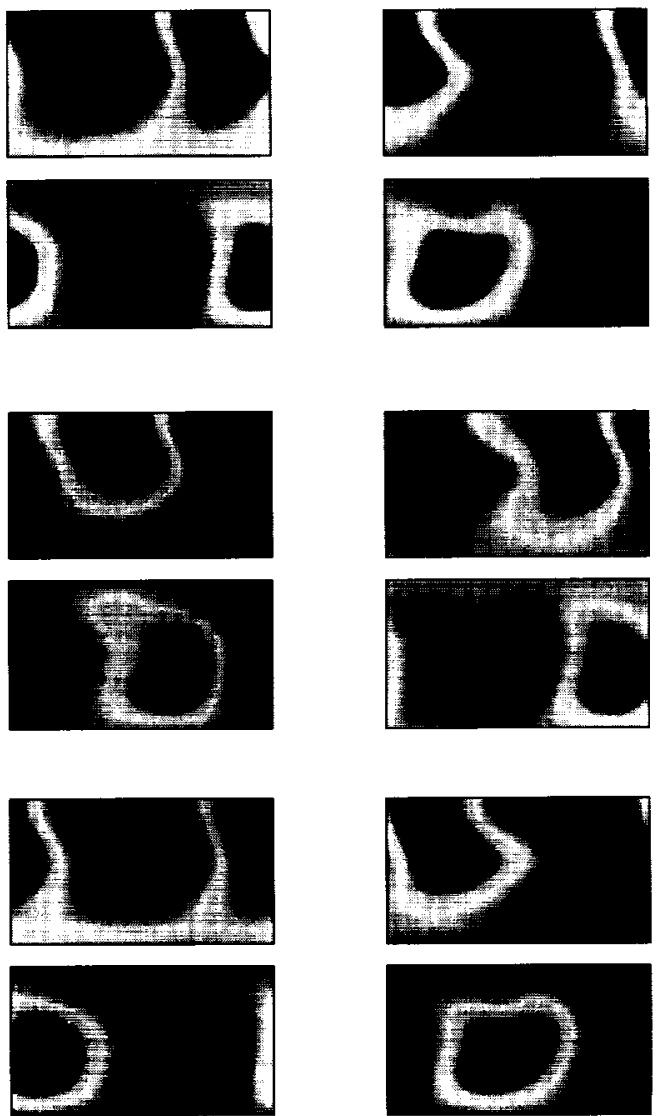

FIGURE 20 Magnetic Field Modulated Waves. ( form of the solutions for A (top) and B (boltom) as time progresses for $D=-400$. (See Color Plate VII

and fifth snapshot negative field dominates, whilst in the third the positive field is stronger.

Further increase in $|D|$ leads to subsequent tertiary Hopf bifurcation to motion on a two-tours in 'energy-space' (shown in Fig. 21) which breaks down to form the chaotic attractor. For these solutions the spatial structure is complicated. The nodes are now irregular and breathe erratically, as well as travelling quickly to the right. The velocity field behaves in much the same way .. wild oscillations are superimposed on a net propagation of the waves to the right. If the 

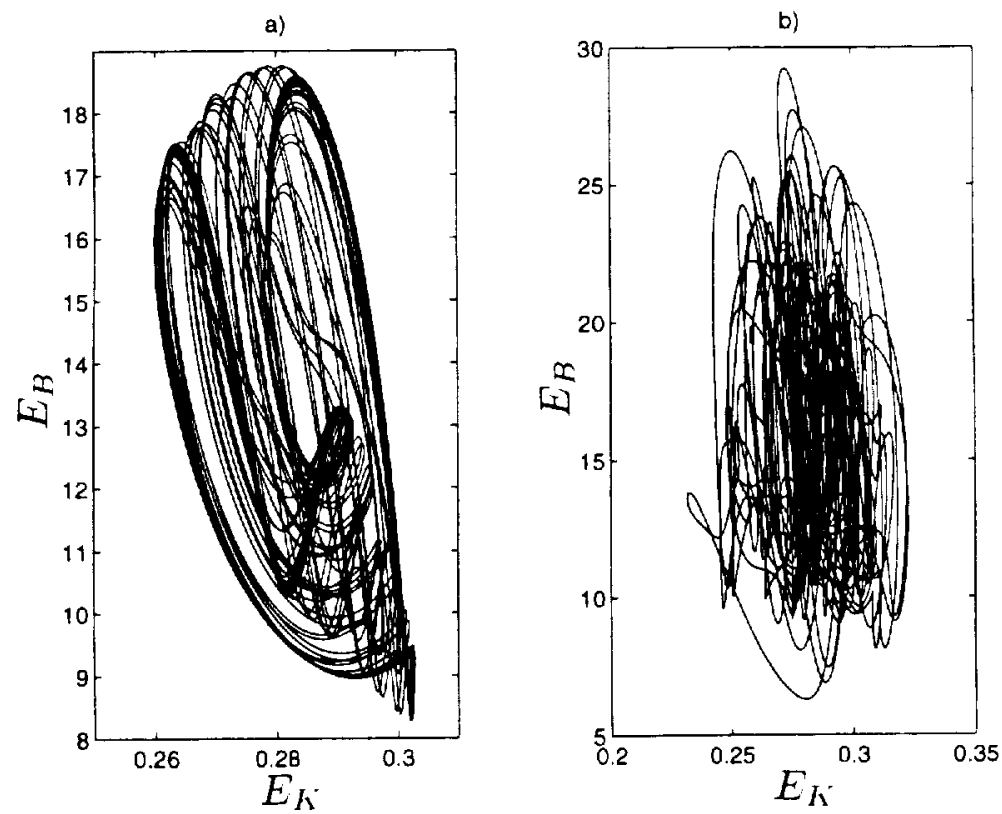

FIGURE 21 Two-torus and chaotic attractor (in constant): Plots of the trajectories in the $\left(E_{B}, F_{K}\right)$ plane for (a) $D$ - 11000 . (b) $D--1500$.

dynamo number is increased further these oscillations become larger in amplitude.

This model therefore undergoes the same sequence of bifurcations as the more realistic model discussed earlier. The travelling waves become unstable in a series of Hopf bifurcations leading to motion on a torus which breaks down, leaving a chaotic attractor. The modulation is therefore a direct result of including the MalkusProctor mechanism and the equation for the large-scale velocity perturbations. Any complicated spatial behaviour is a secondary effect, which is only present because of the bifurcations to modulated waves. (If travelling waves remain stable then the spatial structure stays simple, as in the local quenching models.) Whilst the spatial dependence may enhance the temporal behaviour and encourage the transition to spatio-temporal chaos, it is not an integral part of the mechanism. 


\subsubsection{Changing the Magnetic Prandtl Number}

We now return to the initial Parker model with diffusivity varying across the layer, as this model is more relevant to the generation of fields at the base of the convection zone. The consequence of varying the magnetic Prandtl number on the nature of solutions is investigated. In the low-order model studied by Weiss et al. (1984) and Jones et al. (1985), the bifurcation sequence was fairly sensitive to the choice of the value of the magnetic Prandtl number with the periodic solution remaining stable unless $\tau<1$. Clearly if the same is true for the PDEs investigated here, then this would have serious consequences for the claim that the bifurcation sequence and route to chaos found here are robust and therefore astrophysically relevant.

For this reason, results are now reported for $\tau=0.5$ and $\tau=2$. (Changing the magnetic Prandtl number by an order of magnitude presents problems for the numerical scheme though this is addressed in the next section.) The results for $\tau=0.5$ are similar to those for $\tau=1$. The bifurcation sequence is the same and the only difference is in the value of the dynamo number at which the bifurcations take place. The bifurcations for this model occur earlier (at lower values of $|D|$ ) than for when $\tau=1$. This is to be expected as the smaller the value of $\tau$, the larger the effect of a given magnetic field strength on the velocity perturbations. The time series for the magnetic energy for three types of solution (travelling waves, modulated waves and quasiperiodic waves) are shown in Figure 22.

Not only is the bifurcation structure the same for this parameter value, but the spatial dependence of solutions is similar to that for $\tau=1$ - the two-layer structure of the toroidal field remains, and the four nodes of magnetic field persist in the lower layer. The spatial dependence is therefore the same as that shown in Figure 14.

For $\tau=2$ a similar bifurcation sequence is again observed. This time the bifurcations are delayed in comparison with the $\tau=1$ case. Here though the spatial dependence of the solutions is slightly different. The series of snapshots for the toroidal field for values of the dynamo number that give modulated waves in Figure 23, shows that although there are still two layers of magnetic field, the slow-moving magnetic field (at the bottom of the box) has a similar horizontal scale to that in the fast-moving magnetic field. This can be ascribed to the increase in 

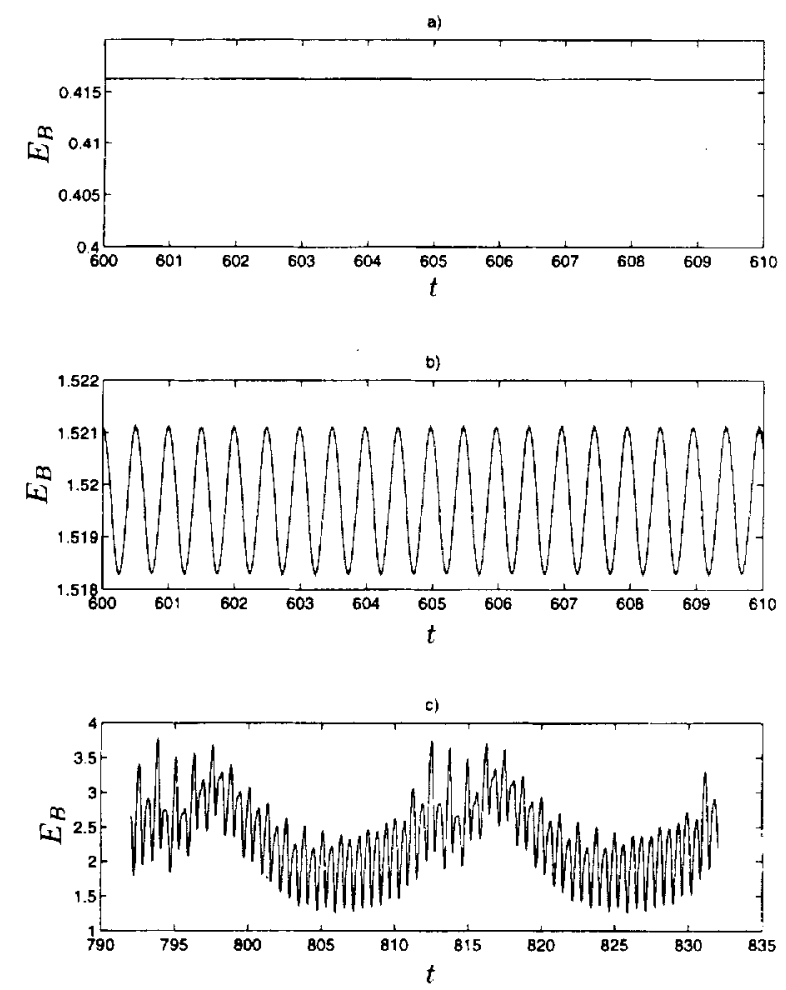

FIGURE 22 Solutions for $\tau=0.5$. Magnetic energies for (a) $D=-50$, (b) $D=-200$, (c) $D=-500$.

$\tau$ leading to a larger scale for the magnetically driven velocity perturbations. Apart from the scale of the field and velocity perturbations, the solutions are similar to those for $\tau=1$, and the sequence of bifurcations is certainly the same - a succession of Hopf bifurcations followed by breakdown of a tours leading to spatio-temporal of chaos. The spatial dependence of the chaotic solutions is slightly different to that for $\tau=1$, but in all other respects the solutions are similar.

\subsubsection{A Note on Very Low Prandtl Numbers}

So far in this section only $\mathrm{O}(1)$ values of $\tau$ have been considered. As noted in the previous section, changing the value of the magnetic 

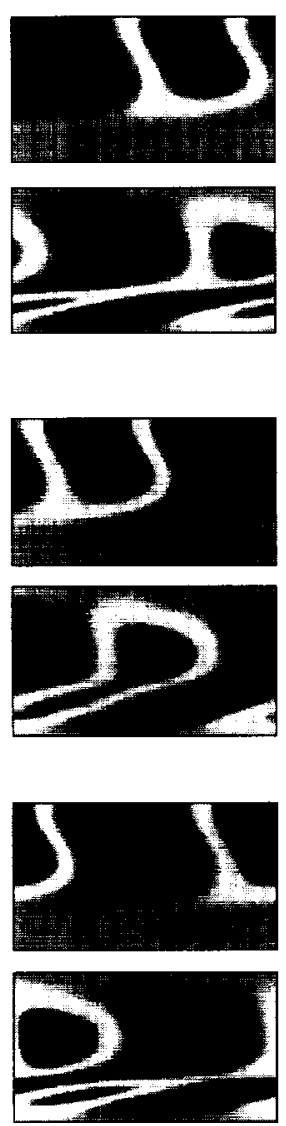
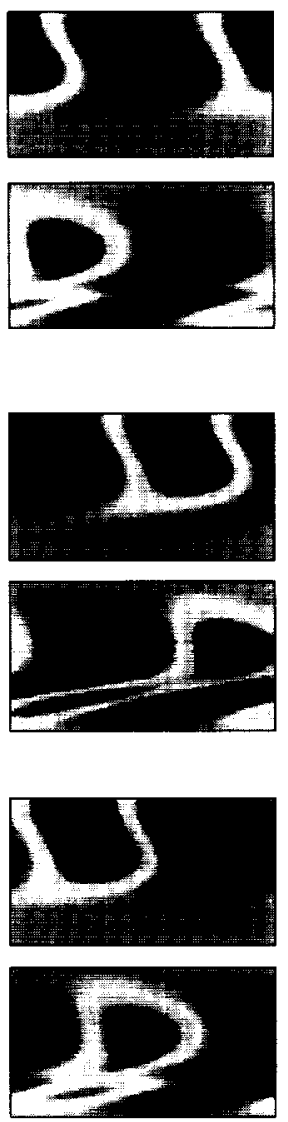

FlgURE: 23 Magnetic field Modulated waves for $\tau \cdot$ 2: As for Figure 14, but $T \cdot 2$ atnd $l^{-}-250$. This time the magnetic field generated by the velocity perturbations has the same horizontal scale as that generated by the imposed shear. (See Color Plate VIII).

Prandtl number by an order of magnitude presents problems for the numerical scheme in the model where the diffusivity is a function of position. However the effects of very low Prandtl numbers can be examined in the constant-11 model discussed in Section 6.2.2. A thorough investigation of parameter space is certainly not possible in this case, as it takes very many magnetic diffusion times for transients to die away. For this reason only some preliminary results have been obtained. The results of this section have led to investigation of the consequences of including the Malkus-Proctor effect with a low value 
of $\tau$ in a global dynamo model where the variation of parameters with latitude is included (Tobias 1996a, 1997).

Here the magnetic Prandtl number is set to be 0.01 and numerical integrations are performed for a selection of dynamo numbers. We are primarily interested in the behaviour of solutions for large(ish) values of $|D|$. The models already discussed do produce chaotic modulation of the basic magnetic cycle, but this is a weak effect, with the amplitude of modulation small in comparison to the amplitude of the basic cycle. So it is important to ascertain whether the low- $\tau$ limit yields solutions where the magnetic energy is substantially modulated and where periods of greatly reduced activity (corresponding to minima) can be detected.

The first value of the dynamo number investigated was $D=-2100$, which is far in the nonlinear regime. The results are shown in Figure 24. The times series for both the magnetic and kinetic energy are periodic. This indicates that the solutions have undergone both a primary Hopf bifurcation to travelling wave (constant energy) solutions and a secondary Hopf bifurcation to quasiperiodic solutions (with periodic
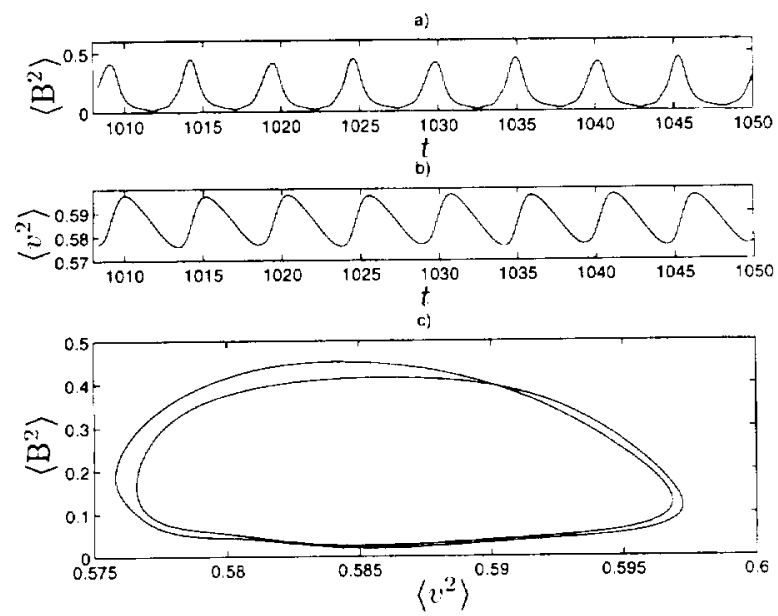

FIGURE 24 Period-2 orbit: Low $\tau$ solutions for $\tau=0.01, D=-2100$. (a) Average magnetic energy as a function of time. It is important to remember that the basic magnetic cycle has been removed from this time series by averaging. The time series therfore gives the amplitude of the modulation. there are episodes when the magnetic energy reduces drastically to zero. (b) As for (a), but for the average kinetic energy. (c) Phase-plane trajectory inergy space. Graph of $\left\langle B^{2}\right\rangle$ vs $\left\langle v^{2}\right\rangle$. 
average energies). Apparently the solution has also undergone a subsequent period-doubling bifurcation. The figure clearly shows that the modulation of the basic cycle (which has been removed by the averaging procedure) is a large effect and that there are episodes when the magnetic energy is very small.

Chaotic modulation can be found if the magnitude of the dynamo number is increased further, as in Figure 25 where solutions for $D=-2500$ are shown. The kinetic and magnetic energy are both irregularly modulated and the attractor in phase space appears to be chaotic. The modulation is still a large effect and many episodes of reduced activity are detected here.

These results give an indication of how solutions behave if the magnetic Prandtl number is reduced by an order of magnitude. It seems a though the low- $\tau$ limit leads to significant amplitude modulation of the basic magnetic activity. The low value of the Prandtl number makes larger velocity perturbations easier to excite, thereby increasing the amplitude of the modulation.
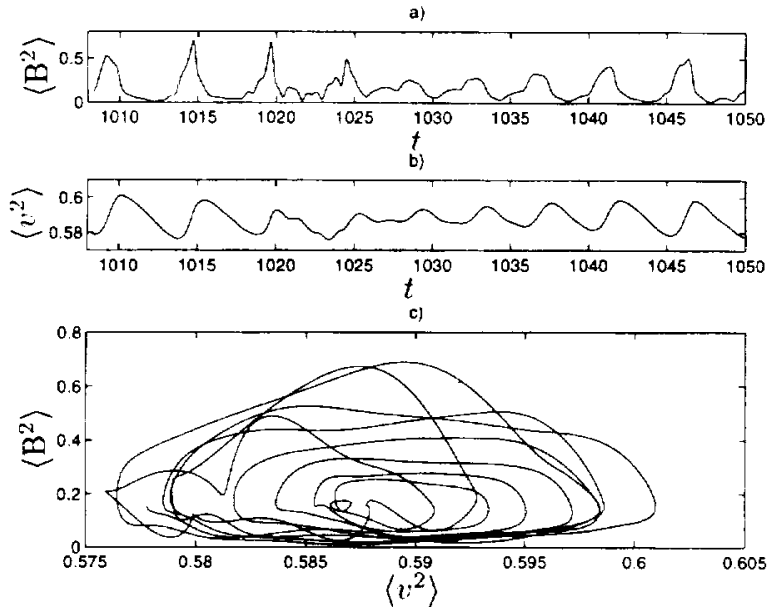

FIGURE 25 Chaotic orbit: Lot $\tau$ solutions for $\tau=0.01, D=-2500$. (a) Average magnetic energy as a function of time. (b) As for (a), but for the average kinetic energy. (c) Phase-plane trajectory in energy space. Graph of $\left\langle b^{2}\right\rangle$ vs $\left\langle v^{2}\right\rangle$. Both the magnetic and kinetic energy are now chictically modulated. 


\subsection{Summary for Malkus-Proctor Nonlinearities}

The coupling of the dynamo equations to the mean momentum equation has been studied in two separate cases. In the first problem, velocity perturbations that were independent of $z$ were driven by the averaged Lorentz force. This system produced travelling wave solutions that were stable for all values of the dynamo number $D$ and in this respect the solutions were the similar to those found for the simple quenching mechanisms discussed in Section 5. It is interesting to note that the magnetic field generated velocity perturbations away from the region of strongest magnetic field, differing from the quenching mechanisms where only local action of the magnetic field can be modelled. The wavespeed increased substantially with increasing dynamo number.

Interesting time-dependent behaviour was only achieved if the local field was allowed to interact with the velocity in the mean momentum equation. The important features of the bifurcation sequence are robust to changes in the parameters and even to changes in the profile for the diffusivity. The trivial state becomes unstable to dynamo action in a Hopf bifurcation (which is usually supercritical) to travelling wave solutions. Solutions are steady in a frame moving with the wavespeed and the average magnetic and kinetic energy are constant. These travelling waves lose stability in a secondary Hopf bifurcation as the dynamo number is further increased - the average energies now oscillate about a mean value. A further (tertiary) Hopf bifurcation leads to motion on a two-tours in 'average energy space' (or a threetorus in 'magnetic field space'). Solution that exhibit spatio-temporal chaos are found as the dynamo number is increased further and the torus breaks down. It is also possible to find other bifurcations (pitchfork and gluing bifurcations) if the parameters are chosen carefully, but these are not essential parts of the bifurcations to chaotic solutions. Complicated spatial dependence of solutions is also found but this is a secondary effect. The most significant modulation was obtained when the magnetic Prandtl number $(\tau)$ was set to be small.

The route to chaos is therefore exactly the same as that found in the sixth-order model of Weiss et al. (1984). In coordinates moving with the wavespeed, the initial Hopf bifurcation becomes a pitchfork and the bifurcation structure is now the same as the simpler model of Jones 
't al. (1985). It is important to understand why the system must undergo three Hopf bifurcations before chaotic behaviour can be found. The frequency introduced in the Hopf bifurcation to travelling waves is removable (as described above) and if this frequency is removed the travelling waves can be thought of as steady solutions. This was noted by Ruelle (1973) for waves in a rotating system (but the extension to periodic systems is immediate). With the frequency removed, the fixed point must undergo two further Hopf bifurcations before frequency-locking and the formation of a chaotic attractor via the breakdown of a torus can occur (Rand, 1982). So here the route to chaos is slightly different from that for the case where the system has boundaries in Tobias (1996a). There the initial Hopf bifurcation was not to travelling waves, but to genuinely periodic solutions and the frequency of oscillation was not removable. Therefore only one more Hopf bifurcation was required to introduce the possibility of frequency-locking and breakdown of the torus.

It is therefore only due to the artificial nature of the periodic boundary conditions that the system must undergo a tertiary bifurcation before chaotic solutions may be found. In systems with more realistic boundary conditions this may occur after only two Hopf bifurcations and this is therefore the genetic scenario for transition to chaos in these dynamo models.

\section{CONCLUSION}

The explanation of the origin of magnetic activity in the sun and stars provides a challenge to the theoretician. In the past this challenge has been met in a rather ad-hoc and haphazard way. Many nonlinear dynamo models have been proposed and results exhibited, but relatively little has been learned of the underlying structure of nonlinear dynamos. The models have only scraped the surface of the problem - the results are clear, but what are their consequences for stellar magnetic field generation? Is the behaviour found model-dependent, and if not why is it a robust feature of the dynamo problem?

By carrying out a systematic investigation of a simple dynamo model, I have attempted to provide a clear and coherent picture of the 
rôle of nonlinear effects in mean-field dynamos. Given that there is so much that is poorly understood about the nature of the dynamo problem, it is only by interpreting the results in the light of the recent advances in dynamical systems theory that progress may be made. We need to separate out the robust behaviour and discard any results that are model-dependent. An understanding of the fundamental properties of nonlinear systems is essential in order to achieve this.

Local travelling solutions do yield a greater understanding of the consequences of nonlinearities for the radial dependence of solutions. Moreover, much can also be learned about the consequences of including simple quenching mechanisms. Quenching of the turbulent diffusion is found to have a more subtle effect than either $\alpha$ or $\omega$ quenching, as it modifies the spatial structure of solutions. However, the unrealistic choice of ( periodic) lateral boundary conditions for this local model implies that any global properties (e.g., wavespeed, frequency) of such a dynamo may be misleading (Tobias et al.. 1997). This is an important result, as it demonstrates that over-simplified models may lead to incorrect conclusions about the behaviour of dynamos.

Quenching mechanisms, although simple to implement, are unrealistic parameterizations of the back-reaction of the magnetic field on the velocity. These mechanisms are instantaneous in time and local in space a severe restriction on the possible consequences of including nonlinearities. It is interesting to note that, because of the simplicity (and local nature) of the quenching mechanisms often used in nonlinear mean field dynamos, the behaviour of the solutions is not critically dependent on the precise form of the quenching chosen. This was shown to be the casse as the imposition of both 'averaged' (oncdimensional) and 'local' (two-dimensional) quenching mechanisms yielded similar results.

Increasingly complicated spatio-temporal behaviour may be achieved in a natural way by including an equation for the mean velocity perturbation driven by the magnetic field (the Malkus-Proctor effect) as in Section 6. This nonlinearity is more complicated than the simple prescribed quenching formulae for two reasons. The first is that this nonlinearity is non-local in space. The velocity perturbations are not driven where the field is largest but where the gradient of the poloidal and toroidal fields interact. Hence, for this nonlinearity, the 
results are sensitive to the choice of averaged or local interaction. Averaged interaction yielded results similar to those for the quenching mechanisms already discussed, with travelling waves remaining stable. However, if local interactions are allowed then the system displays very different behaviour with the travelling waves becoming unstable to solutions with more complicated time-dependence.

Moreover the torsional oscillations observed in the sun can be attributed to such a velocity perturbation. The addition of a separate evolution equation then allows for a time-lag between the generation of the field and its effect on the velocity field. This time-lag is controlled by the magnetic Prandtl number $\tau$. It is important to note that the route to chaos is via a succession of Hopf bifurcations and the breakdown of a torus - similar to that for the low-order model of Tobias et al. (1995). This is a very important result as it implies that this mechanism is a robust feature of including the interaction of the magnetic field with the velocity in a realistic way.

What do the above results imply for modelling dynamo action in the sun and other stars? By studying the rôle of nonlinearities in isolation, one can interpret the results of numerical simulations which include latitudinal dependence of parameters and realistic boundary conditions. Indeed the results included in this paper have already been used (Tobias 1996a,1997) to construct global models of the solar dynamo. In those models the low- $\tau$ limit was successfully exploited to investigate the nature of Grand Minima, and to ascertain the relative importance of modulational mechanisms (i.e., the Malkus-Proctor mechanism and Parity interactions) on solar dynamo models. The results are promising but further investigations are required to understand the nature of the interaction of turbulent convection and magnetic fields so that more realistic models may be constructed in the future.

\section{Acknowledgement}

I would like to thank the numerous people who assisted with this paper. In particular I would like to thank Nigel Weiss for suggesting the problem. I have also benefitted from discussions with Keith Moffat, Mike Proctor and Andrew Soward. The work was instigated whilst I was a PHD student at the University of Cambridge under a 
PPARC studentship and completed at JILA under NASA SPTP grant NAG5-2256.

\section{References}

Beer, J. Raisbeck, G. M. and Yiou, F., "Time variations of ${ }^{10} \mathrm{Be}$ and solar activity," in: The Sun In Time (Ed. C. P. Sonett, M. S. Giampapa, and M. S. Matthews,), pp. 343-359. University of Arizona Press, Tucson. (1991).

Brandenburg, A., Krause, F., Meinel, R., Moss, D. and Tuominen, 1., "The stability of nonlinear dynamos and the limited role of kinematic growth rates," Astron. Astrophys. 213, $411-422$ (1989).

Cattaneo, F. and Hughes, D. W., "Nonlinear saturation of the turbulent $\alpha$-effect," Phys. Rev. E54, R4532-R4535 (1996).

Charbonneau, P. and Macgregor, K. B., "On the generation of equipartition-strength magnetic fields by turbulent hydromagnetic dynamos," Astrophys. J. Lett (submitted) (1996).

Choudhuri, A. R., "Stochastic fluctuations of the solar dynamo," Astron. Astrophys. 253, $277-285$ (1992).

Covas, E., Tworkowski, A., Brandenburg. A. and Tavakol, R., "Dynamos with different formulations of the dynamic $\alpha$-effect," Astron. Astrophys. 317, 610 (1997).

Eddy, J. A., "The Maunder minimum," Science 192, 1189 - 1202 (1976).

Gilman. P. A., "Dynamically consistent nonlinear dynamos driven by convection in a rotating spherical shell. II. Dynamos with cycles and strong feedbacks," Astrophy's. J. Suppl. Ser. 53, 243-268 (1983).

Glatzmaier, G. A., "Numerical simulations of steller convective dynamos 2. Field propagation in the convection zone," Astrophys. J. 291, 300-307 (1985).

Gough, D. O., Moore, D. R., Spiegel, E. A. and Weiss, N. O., "Convective instability in a compressible atmosphere II," Astrophys. J. 206, 536-542 (1976).

Hoyng, P., "Turbulent transport of magnetic fields. III stochastic excitation of global modes," Astrophys. J. 332, 857-871 (1988).

Jones. C. A., Weiss, N. O. and Cattaneo, F., "Nonlinear dynamos - a complex generalization of the Lorenz equations," Physica 14D, $161-176$ (1985).

Kitchatinov, L. L., Rüdiger, G. and Küker, M., " $\Lambda$-quenching as the non-linearity in stellar turbulence dynamos," Astron. Astrophys. 292, 125-132 (1994).

Kleeorin. N. I. and Ruzmaikin A. A., "Properties of a nonlinear solar dynamo model," Geophys. Astrophys. Fluid Dynam. 17, $281-296$ (1981).

Knobloch, E. and Landsberg, A. S., "A new model of the solar cycle," Mon. Not. R. ast. Soc. 278, 294 (1996)

Malkus, W. V. R. and Proctor, M. R. E., "The macrodynamics of the alpha-effect in rotating fluids," J. Fluid Mech. 67, 417--444 (1975).

Meunier, N., Proctor, M. R. E., Sokoloff, D. D., Soward, A. M. and Tobias, S. M. "Asymptotic properties of a nonlinear $\alpha \omega$-dynamo wave: period, amplitude and latitude dependence," Geophys. Astrophys. Fluid Dynam. 86, 249- 285 (1977).

Noyes, R. W.. Wiess, N. O. and Vaughan, A. H., "The relation between steller rotation rate and activity cycle periods," Astrophys. J. 287, 769-773 (1984)

Ossendrijver, A. J. H. and Hoyng, P., "Stochastic and nonlinear fluctuations in a mean field dynamo," Astron. Astrophys. 313, $938-948$ (1996).

Parker, E. N., "Hydromagnetic dynamo models," Astrophys. J. 122, $293-314$ (1955).

Parker, E. N., Cosmical Magnetic Fields: Their Origin and Activity. Clarendon Press, Oxford. (1979).

Parker, E. N., "A solar dynamo surface-wave at the interface between convection and nonuniform rotation," Astrophys. J. 408, 707-719 (1993). 
Platt, N., "On-off intermittency: General description and feedback model," in: Theory of Solar and Planetary Dynamos (Ed. M. R. E. Proctor, P. C. Matthews, and A. M. Rucklidge,), pp. 249 - 264. Cambridge University Press, Cambridge. (1994).

Platt, N., Spiegel, E. A. and Tresser, C., "The intermittent solar cycle," Geophys. Astrophys. Fluid Dynam. 73, 147 - 161 (1994).

Rand, D.. "Dynamics and symmetry: Predictions for modulated waves in rotating fluids," Arch. for Rational Mech. Anal. 79, 137 (1982).

Ribes, J. C. and Nesme-Ribes, E., "The solar sunspot cycle in the Maunder minimum AD 1645 - AD 1715," Astron. Astrophys. 276, 549563 (1993)

Roberts, P. H. and Soward, A. M., "A unified approach to mean field electrodynamics," Astron. Nachr. 296, 49-64 (1975).

Ruelle, D., "Bifurcations in the presence of a symmetry group," Arch. for Rational Mech. Anal. 51, 136-152(1973).

Schmalz, S. and Stix, M., "An $\alpha \Omega$ dynamo with order and choas," Astron. Astrophys. 245, 654-661 (1991).

Schmitt, D., Schüssler, M. and Ferriz-Mas, A., "Intermittent solar-activity by an on-off dynamo," Astron. Astrophys. 311, Ll-L4 (1996).

Spiegel, E. A., "The chaotic solar cycle," in: Lectures in solar and planetary dynamos (Ed. M. R. E. Proctor and A. D. Gilbert), pp. 245-266. Cambridge University Press. Cambridge (1995).

Steenbeck, $M$. and Krause, F.. "Zur Dynamotheorie stellarer und planetarer Magnetfelder I. Berechnung sonnenähnlicher Wechselfeldgeneratoren," Astron. Nachr. 291, $49 \cdot 84$ ( 1969).

Stix, M., "Non-linear dynamo waves," Astron. Astrophys. 20, $9-12$ (1972)

Stuiver, M., "Atmospheric $\mathrm{C}^{14}$ as a proxy of solar and climate change," in : The Solar Engine and its influence on terrestrial atmosphere and climate (Ed. Nesme-Ribes, E.), pp. 203-220. Springer-Verlag, Berlin Heidelberg New York (1994).

Thompson, M. J.. Toomre, J., Anderson, E. R.. Anita, H. M., Berthmicu, G., Burtonclay, D., Chitre, S. M., Christensen-Dalsgaard, J., Corbard, T., DeRosa, M., Genovese, C. R.. Gough, D. O., Haber, D. A., Harvey, J. W., Hill, F., Howe, R., Korzennik, S. G., Kosovichev, A. G., Leibacher, J. W., Pijpers, F. P., Provost, J., Rhodes Jr., E. J., Schou, J., Sekii, T., Stark, P. B. and Wilson, P. R., "Differential rotation and dynamics of the solar interior," Science 272, 1300-1305 (1996).

Tobias, S. M., "Grand Minima in nonlinear dynamos," Astron. Astrophys. 307, L21 L24 (1996a)

Tobias, S. M., "Diffusivity quenching as a mechanism for Parker's surface dynamo." Astrophys. J. 467, $870-880$ (1996b).

Tobias, S. M., "The Solor Cycle: parity interactions and amplitude modulation," Astron. Astrophys. (submitted) (1997).

Tobias, S. M., Proctor, M. R. E. and Knobloch, E.. "The rôle of absolute instability in the solar dynamo," Astron. Astrophys. 318, L55 L58 (1997).

Tobias, S. M., Weiss, N. O.. and Kirk, V., "Chaotically modulated stellar dynamos," Mon. Not. R. ast. Soc. 273, 1150 - 1166 (1995).

Vainshtein, S. I. and Cattaneo, F., "Nonlinear restrictions on dynamo action," Astrophys. J. 393, $165-171$ (1992).

Watari, S., "Chaotic behaviour of the north-south asymmetry of sun-sports?" Solar Phys. 163, 617-625 (1996).

Weiss, N. O., "Bifurcations and symmetry-breaking in simple models of nonlinear dynamos," in: The Cosmic Dynamo (Ed. F. Krause, K.-H. Rädler and G. Rüdiger), pp. 219-229. IAU Symposia No.157, Kluwer (1993).

Weiss, N. O. and Tobias, S. M., "Modulation of solar and stellar activity cycles," in: Solar and Heliospheric Plasma Physics (Ed. G. M. Simnett), Springer Lecture Notes in Physics, (to appear) (1997)

Weiss, N. O., Cattaneo, F. and Jones, C. A., "Periodic and aperiodic dynamo waves," Geophys. Astrophys. Fluid Dynam. 30, 305-341 (1984) 
Worledge, D., Knobloch, E., Tobias, S. M. and Proctor, M. R. E.. "Dynamo waves in semi-infinite and finite domains," Pros. R. Soc. Lond. A 453, 119143 (1997)

Yau, K. K. C.. "Analysis of pre-telescopic and telescopic sunspot observations," in: Secular solar and geomagnetic variations in the last 10,000 ) years (Ed. F. R. Stephenson and A. W. Wolfendale), pp. 245 - 266. Kluwer, Dordrecht (1988).

Yoshimura, H., "A model of the solar cycle driven by the dynamo action of the global convection in the solar convection zone," Astrophys. J. Stupply. Ser. 29, 467 (1975). 


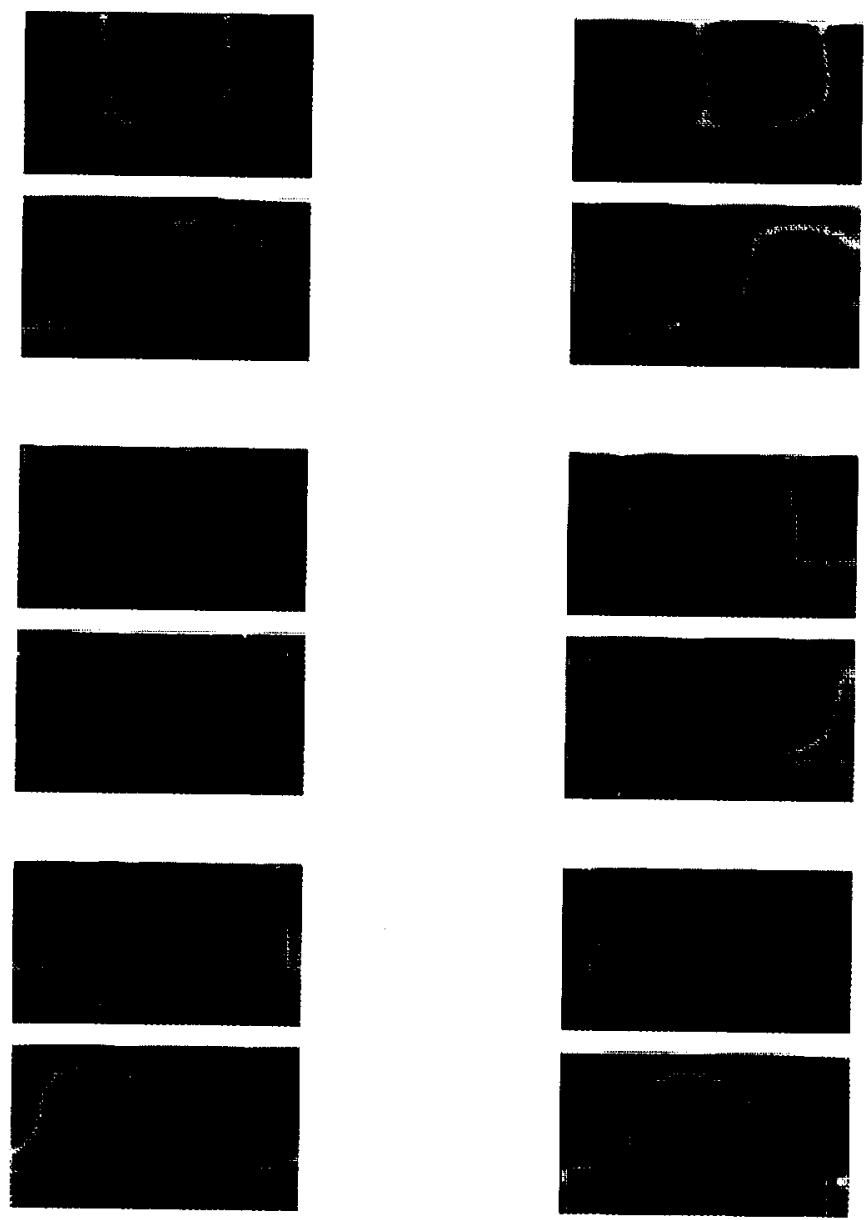

COLOR PLATE I (See S. M. Tobias, page 320). 

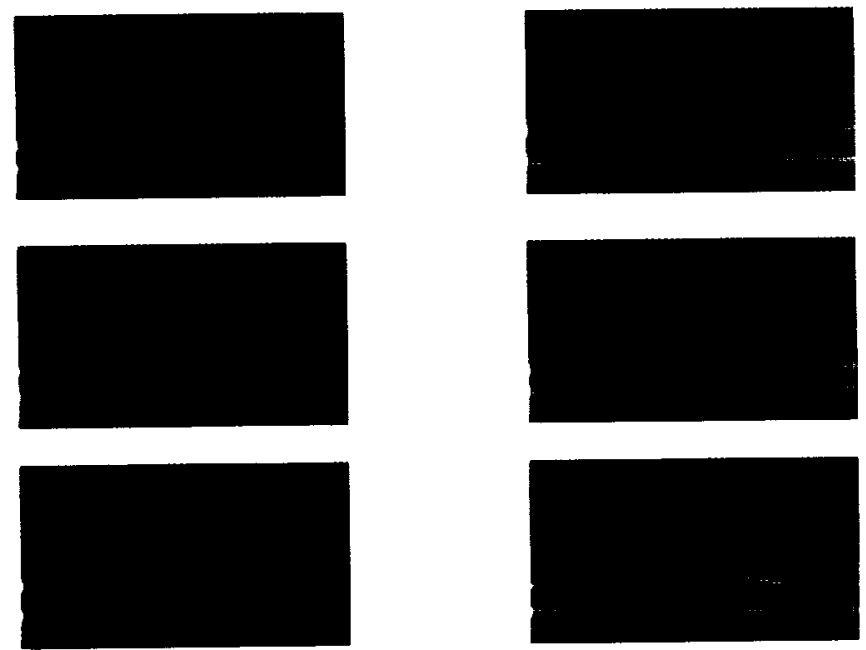

COLOR PLATE II (See S. M. Tobias, page 321). 

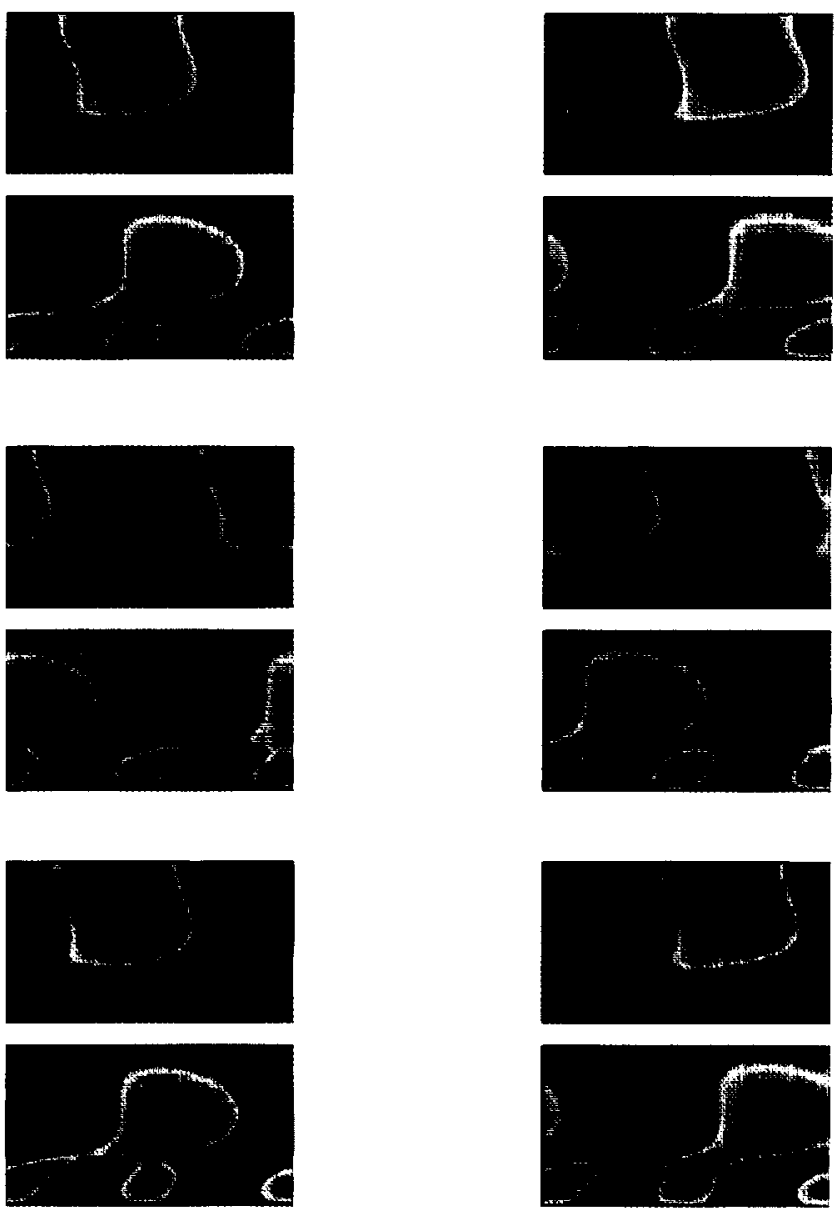

COLOR PLATE III (See S. M. Tobias, page 323). 

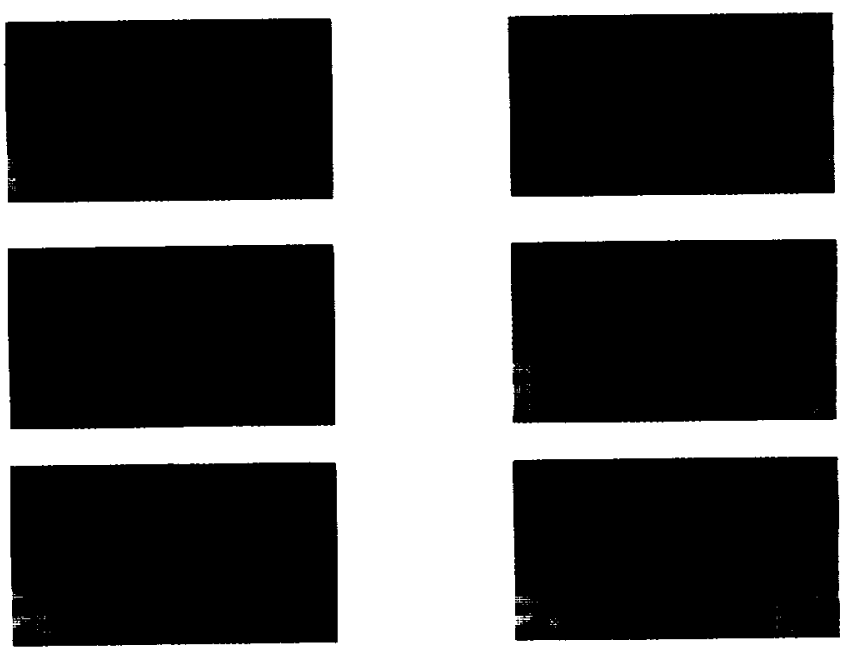

COLOR PLATE IV (See S. M. Tobias, page 324). 

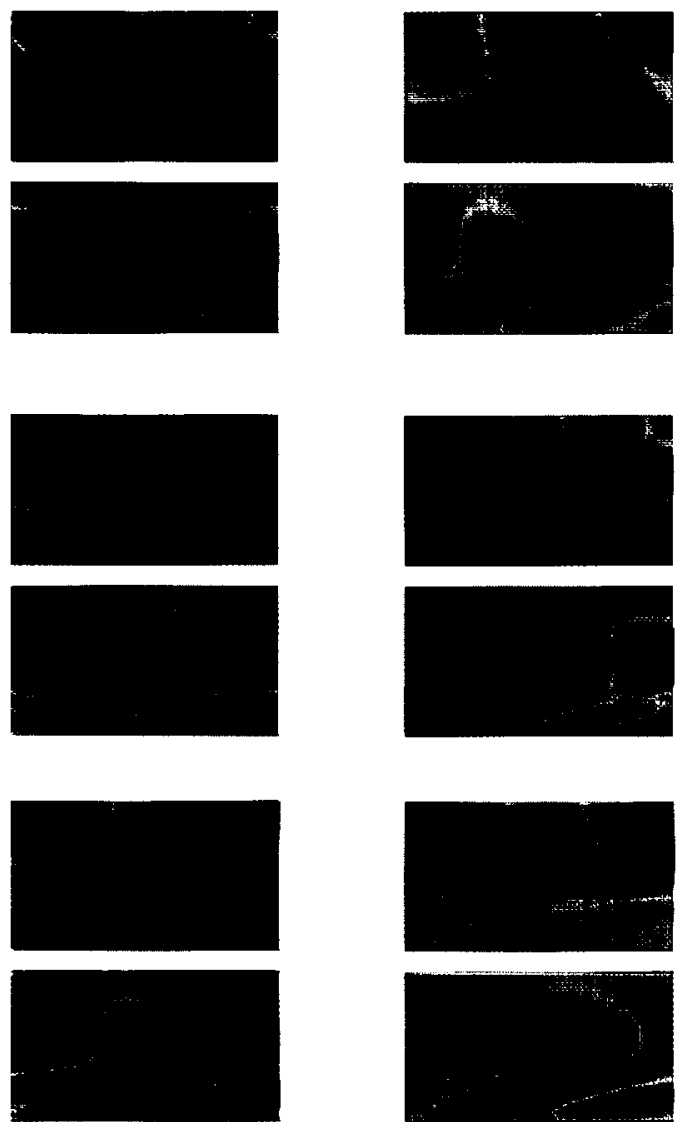

COLOR PLATE V (See S. M. Tobias, page 328). 

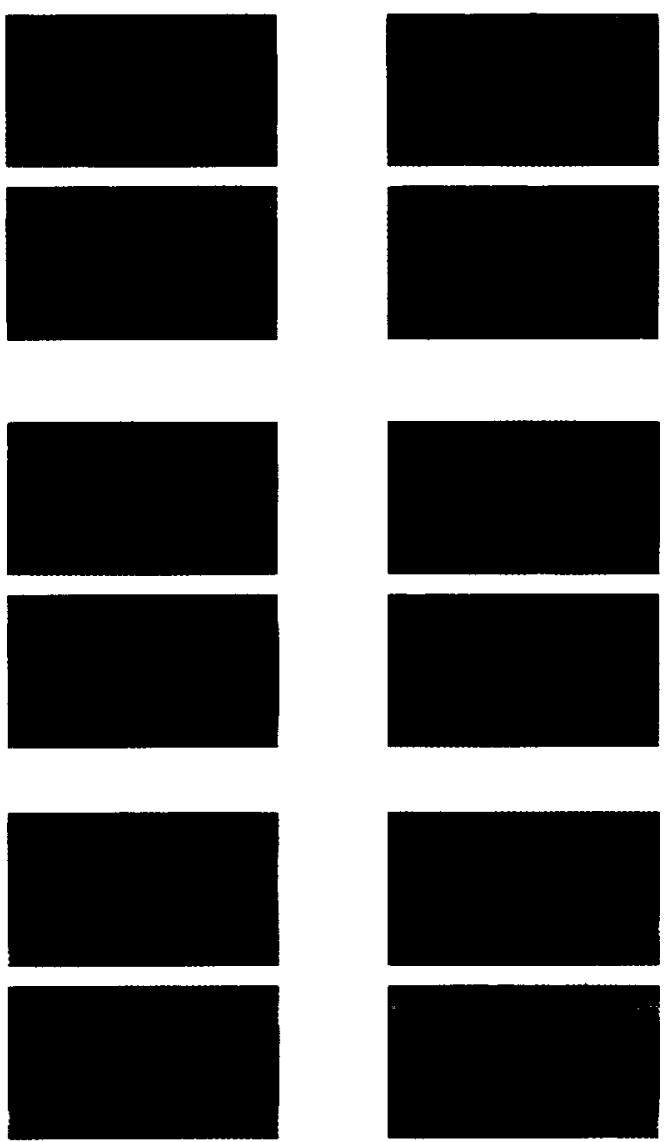

COLOR PLATE VI (See S. M. Tobias, page 329). 

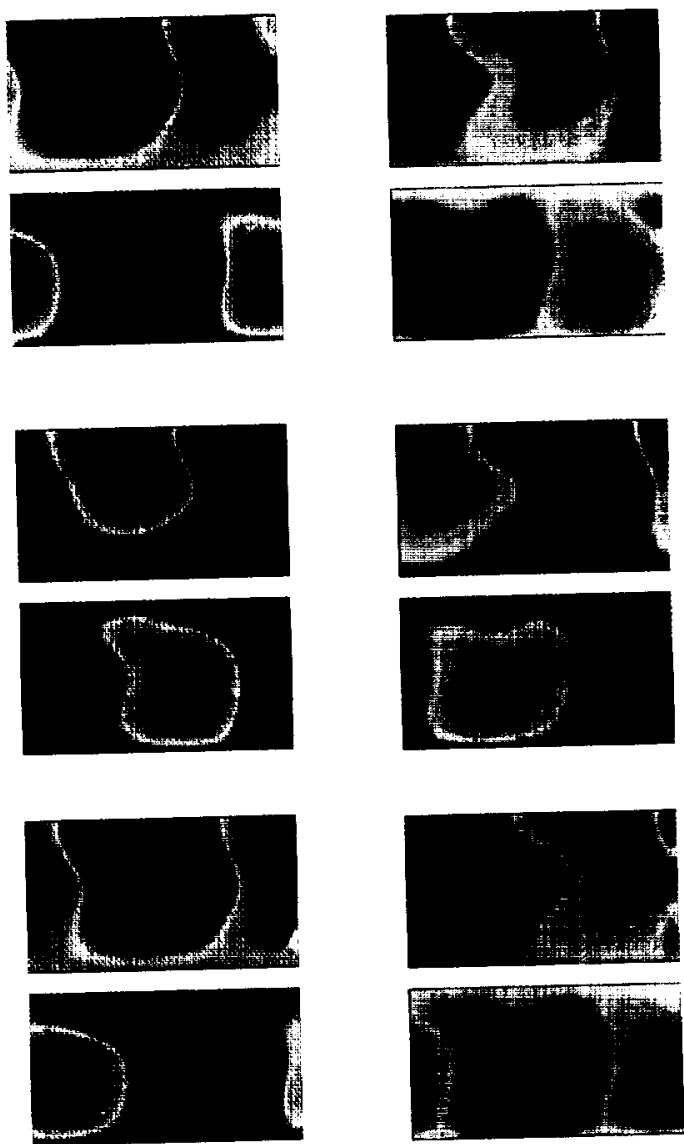

COLOR PLATE VII (See S. M. Tobias, page 330) 

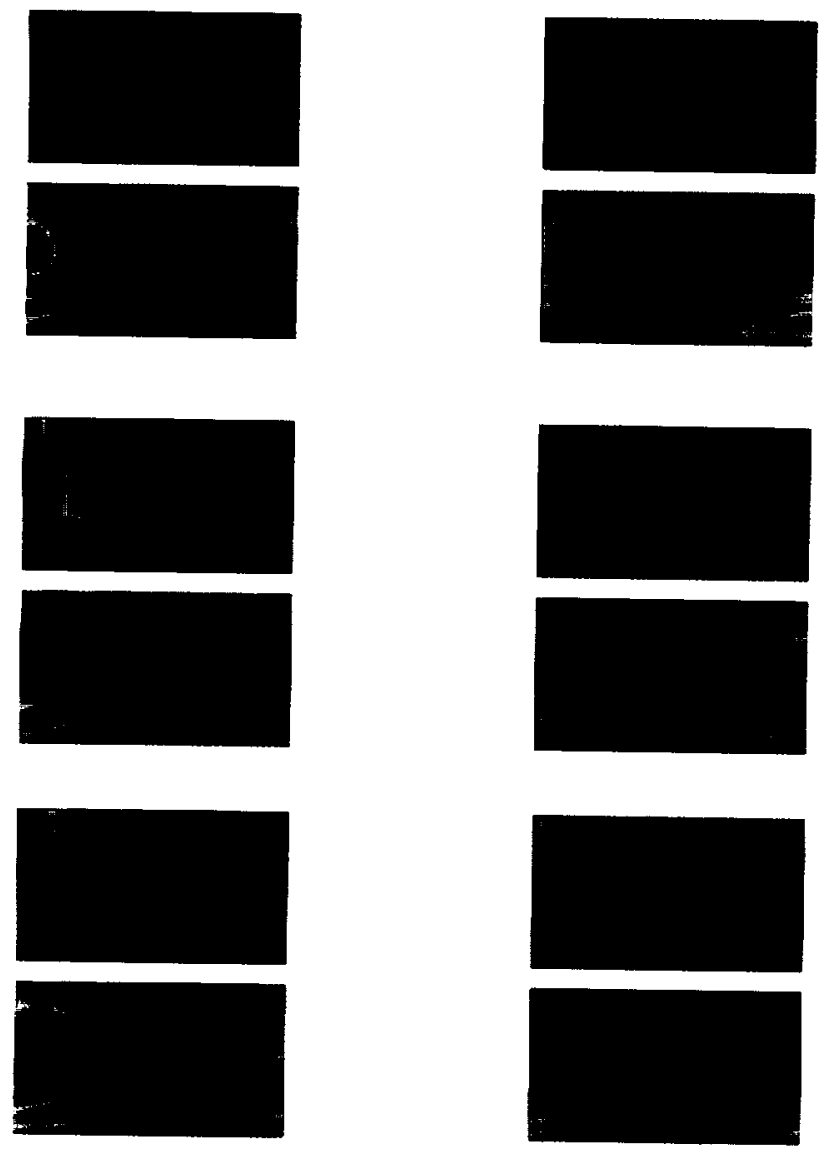

COLOR PLATE VIII (See S. M. Tobias, page 334) 